\title{
La utilización de las revistas electrónicas en la Universidad de León (España): hábitos de consumo y satisfacción de los investigadores
}

\author{
Blanca Rodríguez Bravo \\ María Luisa Alvite Díez * \\ Isabel Olea Merino **
}

Artículo recibido:

8 de noviembre de 2013.

Artículo aceptado:

30 de abril de 2014.

\section{Resumen}

Estudio del comportamiento, preferencias y nivel de satisfacción de la comunidad académica de la Universidad de León con respecto al uso de las revistas electrónicas. Con la finalidad indicada, se analizan los datos y opiniones de los investigadores de 69 áreas de conocimiento, recogidas en encuestas realizadas en 2012. Los resultados obtenidos se contrastan con análisis previos llevados a cabo por el equipo de investigación a partir de estadísticas de descargas y del análisis de datos de productividad. Se constata una frecuencia de uso intensa orientada fundamentalmente hacia las

* Ambas autoras pertenecen a la Universidad de León, España. (blanca.rodriguez@ unileon.es); (luisa.alvite@unileon.es)

**El Profesional de la Información. epi.iolea@gmail.com

INVESTIGACIÓN BiBLIOTECOLÓGICA, Vol. 29, Núm. 66, mayo/agosto, 2015, México, ISSN: 0187-358X. pp. 17-55 
tareas investigadoras. La media de artículos anuales consultados es considerable y manifiesta diferencias claras entre las áreas de humanidades, ciencias sociales y ciencias de la naturaleza y la salud. El número de títulos de revistas consultados de modo regular por los investigadores de la Universidad de León se sitúa en una media cercana a 10 , resultado que supera el umbral hallado en otros trabajos.

Palabras clave: Bibliotecas universitarias; Encuestas; España; Hábitos de consumo; Lectura; Revistas electrónicas; Satisfacción de los investigadores; Universidad de León; Uso.

\section{Abstract}

The use of electronic journals at the University of Leon (Spain): consumer habits and researcher satisfaction

Blanca Rodríguez-Bravo, María-Luisa Alvite-Diez and Isabel Olea-Merino

This study examines the behaviors, preferences and degree of satisfaction of the academic community of the University of Leon with regard to the use of electronic journals. Data and opinions from faculty undertaking research in sixty-nine fields of knowledge were gathered by means of survey applied in 2012. Results were examined against those gathered in an analysis performed by the research team of download statistics and productivity data. Frequent, intense use of online journals is evident largely in the area of research tasks. The average number of articles consulted per year was significant, with marked differences between the fields of Humanities, Social Sciences, and Life and Health Sciences. The average number of journal titles regularly consulted by faculty at the University of Leon was comes nearly to ten, a result that exceeds the average found in other studies.

Keywords: Academic Libraries; Electronic Journals; Habits of Consumption; Readings; Satisfaction of Researchers; Spain; Surveys; University of Leon; Use. 


\section{INTRODUCCIÓN}

— 1 fin del presente trabajo se circunscribe a la identificación de las tendenEcias emergentes en lo que se refiere al acceso y uso de las revistas electrónicas. La exploración se lleva a cabo desde la perspectiva del usuario por mediación de la recopilación de datos en una encuesta y tratará de localizar los factores críticos que afectan el uso efectivo de los contenidos electrónicos.

Las revistas han sido tradicionalmente el principal canal de comunicación de la información en la mayoría de las disciplinas, especialmente en las de ciencias experimentales. Cuando desean dar a conocer sus hallazgos o estar informados de nuevos conocimientos, tanto los creadores como los consumidores de contenidos recurren a las revistas científicas que se constituyen en un cauce de expresión y de recepción de información imprescindible (Delgado López-Cozar, Ruiz-Pérez y Jiménez-Contreras, 2006).

En los niveles de educación superior los recursos electrónicos se han convertido en elementos fundamentales de un entorno de aprendizaje integrado. Su popularidad obedece principalmente a los beneficios de su utilización para el efectivo y eficiente desarrollo de las tareas de quien los emplea. Sus ventajas se pueden resumir en las siguientes: accesibilidad multiusuario sin restricciones temporales ni espaciales, riqueza y variedad de los recursos disponibles y facilidad de búsqueda a través de distintos motores (Deng, 2010).

El formato electrónico facilita la obtención de datos de uso en mayor medida que las colecciones impresas. Las estadísticas de descargas, análisis de citas, ficheros logs y encuestas han demostrado la migración progresiva del formato papel al electrónico. Brady, McCord y Galbraith (2006) en su estudio sobre la Washington State University señalan que el 94 \% de las revistas se utiliza en formato electrónico.

En las últimas décadas se ha realizado una considerable inversión en la adquisición de recursos electrónicos por parte de las bibliotecas universitarias. El incremento de contenidos electrónicos ha sido especialmente significativo en el caso de las revistas adquiridas mediante compra de paquetes siguiendo el modelo Big deal. Las investigaciones fundadas en estadísticas de uso corroboran la variedad y la amplitud de contenidos demandadas por los usuarios y los beneficios que en este sentido proporciona el modelo de compra consorciada de paquetes.

Resulta obvio que tanto las características del medio electrónico como el incremento en el volumen de información electrónica disponible están afectando al comportamiento informativo de los investigadores y, como sugieren Massad, Brown y Tucker (2011), esto repercutirá en la evolución de la propia investigación científica. 
Los diversos estudios realizados a partir de estadísticas sobre descargas de artículos han permitido constatar que la mayor disponibilidad de revistas electrónicas ha incidido positivamente en su utilización y admite una dispersión muy superior en el consumo. No obstante, los estudios derivados del recuento de datos estadísticos presentan ciertas limitaciones, la principal de las cuales se refiere a que resulta discutible que las descargas equivalgan a lecturas en el $100 \%$ de los casos y, por tanto, impliquen unívocamente consumo. Como señalan Nicholas et al. (2008) las descargas significan accesos, no usos. El mismo Nicholas (2008) afirma que no existen evidencias al respecto que indiquen que todos los documentos descargados se lleguen a leer.

Por la razón expuesta, en los últimos años al análisis de los datos estadísticos proporcionados por los agregadores se han sumado estudios sobre el comportamiento de los usuarios, utilizando el análisis de los ficheros logs y de datos recogidos en encuestas. Se trata de indagar el modo y la frecuencia de acceso a las revistas electrónicas en los hábitos de lectura y en la finalidad de la utilización de los contenidos, bien monitorizando los pasos seguidos por los científicos o bien preguntándoles directamente, ya que como apunta Town (2004) contar no sustituye a escuchar.

Parece confirmarse en los estudios pioneros de Tenopir y King (2002), basados en encuestas a los científicos norteamericanos, que los investigadores no sólo leen más artículos que tiempo atrás sino que los leen de entre un abanico más amplio de títulos.

No obstante, Nicholas (2008) argumenta que los investigadores consultan y descargan más documentos que previamente, en un intento de abarcar un espectro similar de información al que accedían antes de disponer de las contrataciones por paquetes. Sin embargo, el tiempo disponible de lectura no puede extenderse ilimitadamente. En este sentido, se ha observado que se dedica menos tiempo que antes a la lectura de cada artículo y que se tiende a leer completos sólo los artículos de menor longitud (Nicholas y Huntington, 2006).

Generalmente se considera que son los investigadores de ciencias los que más utilizan las publicaciones electrónicas. Investigaciones tempranas han señalado que son los químicos, físicos, biólogos y biomédicos quienes hacen un mayor uso de las revistas electrónicas (Tenopir, 2002, 2003; Rusch-Feja y Siebeky, 1999). Trabajos más recientes confirman estos hallazgos, las investigaciones de Tenopir et al. (2005) subrayan que los principales usuarios de las revistas electrónicas son los científicos de ciencias exactas y de ciencias naturales. Eso parece lógico si consideramos que estas áreas han sido las principales usuarias de las revistas como vehículo de información y de comunicación. 
Consideramos significativas las conclusiones del proyecto de CIBER (2011) en el que se encontraron diferencias considerables entre el número de títulos empleados por los investigadores dependiendo de la disciplina; así, sólo un $11 \%$ de los físicos manifiestan leer 10 o más revistas, mientras que para los químicos este porcentaje asciende a un $53 \%$.

La línea de investigación que indaga sobre la utilización de revistas electrónicas se ha consolidado en la última década. Cabe destacar los estudios de Nicholas, Tenopir, Rowlands, Huntington y Jamali en el ámbito anglosajón (Huntington et al., 2006; Nicholas et al., 2006; Nicholas et al., 2008 y Tenopir et al., 2009). En Europa, fuera del Reino Unido, resultan reseñables los estudios de Gargiulo (2003) y Boukacem-Zeghmouri y Schöpfel (2008). Por último, en España apuntamos los trabajos de Borrego et al. (2007), Borrego y Urbano (2007), Ollé y Borrego (2010), Rodríguez et al. (2008), Rodríguez y Alvite (2011) y Rodríguez, Alvite y Barrionuevo (2012).

El estudio que aquí presentamos, basado en el autoanálisis del comportamiento en la búsqueda de información de los investigadores, proporcionará datos útiles para las bibliotecas universitarias y los editores que les permitirán adaptar y mejorar colecciones y plataformas de distribución de contenidos.

Como señala Deng (2010), para proporcionar mejores servicios al usuario resulta imprescindible entender su comportamiento en el uso de los recursos electrónicos y, principalmente, su percepción, expectativas y experiencia en la utilización eficiente de los recursos y servicios que la biblioteca universitaria le ofrece.

\section{OBjetivos y metodología}

El objetivo general del trabajo se dirige a realizar una primera aproximación a los hábitos de consumo de información electrónica de los científicos de la Universidad de León. Con esta finalidad utilizaremos las opiniones de los investigadores de diferentes campos científicos, recogidas en encuestas, las que nos suministrarán las explicaciones, motivaciones y conocimiento de su nivel de satisfacción que los métodos menos intrusivos de análisis de datos estadísticos y monitorización del comportamiento reflejado en ficheros logs no alcanzan a identificar.

En concreto, el presente trabajo pretende analizar los hábitos de consumo sobre el uso de las revistas electrónicas en la Universidad de León. Se realizará una aproximación al comportamiento de los científicos leoneses de distintos ámbitos disciplinares con respecto a la utilización de las revistas electrónicas. 
Es preciso subrayar que la colección de revistas de la Universidad de León se ha incrementado sustancialmente desde los inicios de la década pasada merced a la contratación de los principales paquetes de revistas electrónicas disponibles en el mercado: Emerald, IEEE/IEE Electronic Library Online, JSTOR, ScienceDirect, SpringerLink, Wiley, entre otros. El volumen total de revistas electrónicas suscritas en 2012 asciende a 18228.

Nuestra intención es recabar las opiniones y consideraciones de los investigadores y contrastar los datos obtenidos con los resultados hallados a partir del análisis de las estadísticas de uso y de los datos de visibilidad de la producción científica que se han obtenido como resultado de investigaciones desarrolladas paralelamente.

Con base en la revisión de la literatura relevante planteamos una serie de interrogantes a las que intentamos dar respuesta:

1. ¿Quién está utilizando y accediendo a las revistas electrónicas?

2. ¿Por qué se usan las revistas electrónicas?

3. ¿Con qué finalidad se están utilizando las revistas electrónicas?

4. ¿Cómo se usan las revistas electrónicas?

5. ¿Desde dónde y cuándo se utilizan las revistas electrónicas?

6. ¿Qué repercusión está teniendo el uso de las revistas electrónicas en la investigación de los profesores?

7. ¿Qué títulos son los preferidos por la comunidad académica?

Considerando las interrogantes señaladas se elaboró una encuesta durante el mes de enero de 2012 (Apéndice 1), que una vez testada por profesores pertenecientes a las áreas implicadas en el estudio y realizados los ajustes consecuentes, se envió por correo electrónico a 348 profesores de las áreas de Ciencias experimentales, de la naturaleza y de la salud (CCEE) y a 295 profesores de Ciencias sociales (CCSS) y Humanidades (CCHH) de la Universidad de León en febrero de 2012. El envío se repitió otras dos veces en el mes de marzo intentando conseguir un número mayor de respuestas.

Habida cuenta de que en los últimos cursos el personal docente e investigador se cifraba en poco más de 900, la encuesta llegó al $70 \%$ del profesorado. Se excluyeron la mayor parte de los profesores de las áreas de ingenierías y los académicos de la Facultad de Derecho.

El cuestionario se dirigió a los docentes e investigadores pertenecientes a los departamentos y áreas de conocimiento listados en la Tabla I (Apéndice 2). El personal docente e investigador seleccionado pertenece mayoritariamente a la Escuela Superior y Técnica de Ingeniería Agraria y a las facultades de Ciencias Biológicas y Ambientales, Ciencias Económicas y Empresariales, 
Filosofía y Letras y Veterinaria. En menor medida, a la Escuela Universitaria de Ciencias de la Salud, la Escuela de Ingenierías Industrial e Informática y la Facultad de Educación.

La encuesta (Apéndice 1) estaba conformada por 25 preguntas, estructuradas en cuatro bloques principales que permitían considerar un buen número de indicadores:

1. Análisis de los datos de procedencia y demográficos.

2. Análisis de los datos referentes a pautas de conducta en el consumo de información electrónica.

3. Análisis de los títulos de revistas de uso prioritario.

4. Comparación entre los títulos de uso prioritario identificados en la encuesta con las revistas electrónicas de más descargas en las principales plataformas contratadas y con mayor visibilidad en bases de datos de producción científica.

\section{RESULTADOS Y DisCUSIÓN}

De las 643 encuestas enviadas se recabaron 135 respuestas que suponen un $21 \%$ del total. El número de encuestas recogidas puede considerarse suficiente para hacer un estudio cualitativo.

Recogeremos en este apartado los resultados más significativos. Los ítems de la encuesta fueron analizados descriptivamente por porcentajes para las variables cualitativas.

\section{Análisis de datos de procedencia y demográficos}

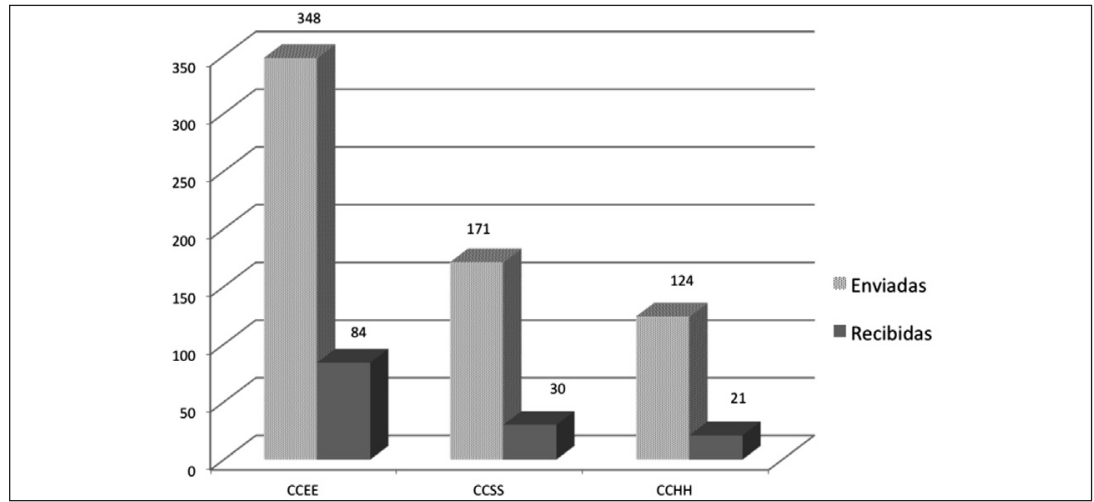

Figura 1. Encuestas enviadas y respuestas obtenidas 
Las respuestas de las áreas de CCEE, 84, suponen un $24.13 \%$ del total de las encuestas enviadas, mientras que en las áreas de CCSS y CCHH la respuesta fue inferior; en concreto, 30 de ciencias sociales que suponen un $17.54 \%$ y 21 respuestas de profesores de humanidades que representan un 16.9\% (Figura 1).

Consideramos que los datos obtenidos reflejan que los usuarios de las revistas electrónicas fueron los más proclives a contestar la encuesta. Asimismo, resulta ampliamente constatado que los científicos de las áreas de ciencias humanas y sociales utilizan en mayor medida los contenidos monográficos y los recursos impresos. Cabe apuntar que esta situación puede atribuirse parcialmente a que las humanidades y las ciencias sociales no se encuentran representadas, en la oferta de contenidos de los proveedores electrónicos, al mismo nivel que las disciplinas de la naturaleza y la salud.

Fueron seis los investigadores que alegaron desconocer y/o no utilizar la oferta de revistas electrónicas de la universidad y sólo contestaron a la pregunta 25. Por tanto, las respuestas a la mayor parte de los ítems se corresponden con 129 investigadores, 81 de ciencias experimentales, 30 de ciencias sociales y 18 de humanidades. Cabe apuntar que todos los científicos de CCSS que han respondido al cuestionario afirman conocer y utilizar las revistas electrónicas disponibles.

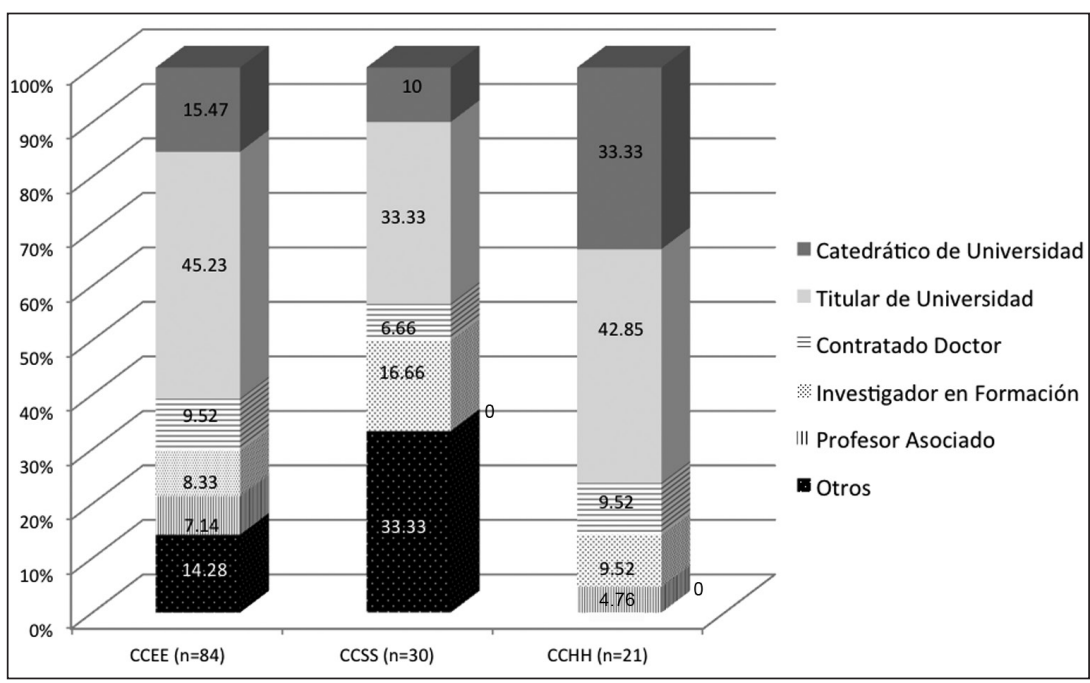

Figura 2. Categorías profesionales

Por categorías académicas (Figura 2), la respuesta más abundante proviene de los Profesores Titulares de Universidad en todas las áreas. Se trata de la categoría más representativa en la Universidad de León y por tanto consti- 
tuye más de un tercio del Personal Docente e Investigador (PDI) total. Los Catedráticos de Universidad son la segunda categoría más numerosa.

El personal en formación engloba ayudantes y las diferentes modalidades de contratos dirigidos al personal de reciente titulación que se encuentra realizando su tesis doctoral.

Minoritarias han sido las respuestas de docentes adscritos a otras categorías como Catedráticos de Escuela Universitaria, Titulares de Escuela Universitaria, Ayudantes Doctores, Profesores Colaboradores, etcétera.

Ha resultado significativo el número de encuestas recibidas de Catedráticos de Universidad en las áreas de humanidades, que han constituido la tercera parte de las respuestas totales recabadas en estas áreas específicamente.

Las respuestas de hombres y mujeres se hallan igualadas, 68 y 67 respectivamente, si bien cabe apuntar que en las áreas de ciencias experimentales predominan las respuestas de los varones (58\%), mientras que en las áreas de ciencias sociales y humanidades priman las de mujeres $(62 \%)$.

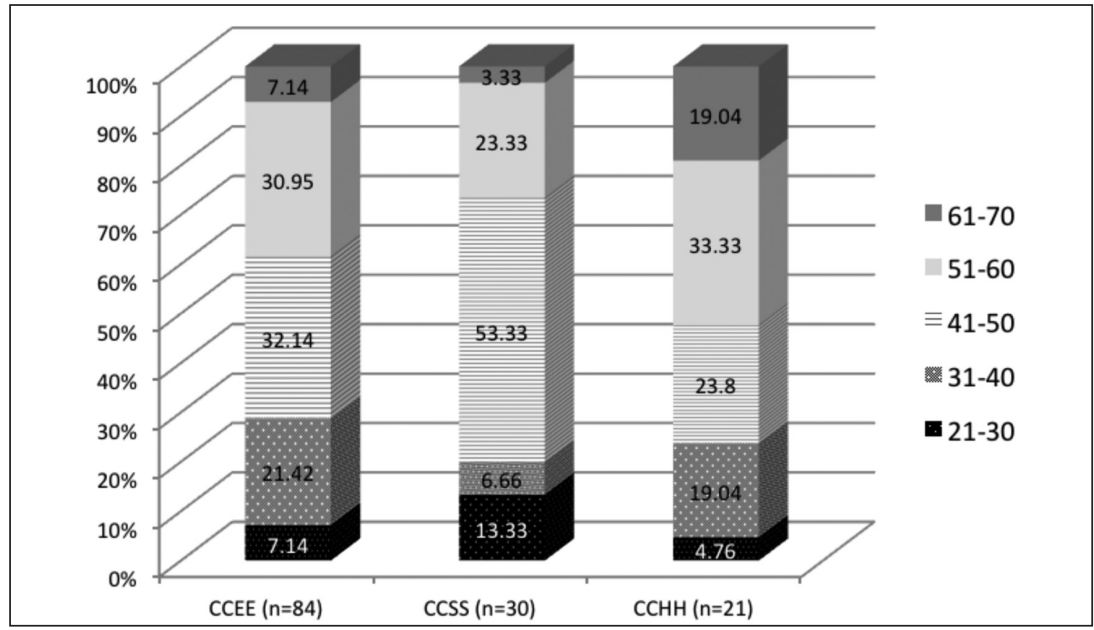

Figura 3. Reparto por edades

Con respecto a los tramos de edad de los que se obtuvieron mayor número de contestaciones, como se observa en la Figura 3, se corresponden con los profesores de edades comprendidas entre 41 y 60 años (65\%). Las franjas de menor incidencia corresponden a los investigadores más jóvenes y de más de 60 años.

No se apreciaron diferencias significativas en los hábitos de consumo de información electrónica entre las categorías profesionales de personal fijo, ni por edad, ni por género. 
No se observa, a partir de los limitados datos obtenidos de la franja de profesores de mayor edad, resistencia a los cambios tecnológicos. Así, cabe apuntar una utilización especialmente intensiva de las revistas electrónicas por parte de los Catedráticos de Universidad, en franjas de edad de entre 51 y 60 años y por encima de los 60 . Este resultado no permite confirmar los hallados en otros estudios como es el de Armstrong et al. (2001) y el de Ollé y Borrego (2010), que identifican a los investigadores más jóvenes como los principales usuarios.

Con carácter general se ha venido considerando a las generaciones últimas como más afines a los recursos electrónicos por su mayor familiaridad con las tecnologías emergentes. Sin embargo, sobre este particular ponen de relieve Nicholas et al. (2007) que las habilidades tecnológicas y los equipamientos no afectan significativamente la utilización de los recursos electrónicos dado que la tecnología web y las herramientas de búsqueda son comunes y fáciles de usar.

En buena lógica, por su dedicación parcial a las labores académicas que se concretan principalmente en tareas docentes, los Profesores Asociados parecen realizar un menor uso de los recursos disponibles.

Consideramos prioritario profundizar en el comportamiento diferenciado de los investigadores en las diversas etapas de la vida académica. Las diferencias de las necesidades de información entre los distintos tipos de investigadores y su comportamiento en la búsqueda de información son incuestionables. Tanto es así que el informe de RLUK (Aukland, 2012) incide en la necesidad de que bibliotecarios especializados temáticamente se ocupen de las necesidades de aprendizaje de los investigadores para cada etapa, entendiendo las diferencias y evaluando cuidadosamente las necesidades individualizadas de los diferentes grupos.

\section{Análisis de los datos referentes a pautas de conducta en el consumo de información electrónica}

El análisis que se presenta a continuación parte de 129 respuestas correspondientes a 81 profesores de ciencias experimentales, de la naturaleza y de la salud; a 30 de ciencias sociales y a 18 de ciencias humanas.

Como resultado general cabe señalar que se aprecia una mayor intensidad de uso entre los investigadores de los departamentos de Biodiversidad y Gestión Ambiental, Biología Molecular, Higiene y Tecnología de los Alimentos y Medicina, Cirugía y Anatomía Veterinaria que entre los del PDI de los cinco departamentos restantes de las áreas de ciencias experimentales. Se aprecia también una superior intensidad de uso entre los investigadores de ciencias sociales con respecto de aquellos de humanidades. 
La vinculación entre el uso de las revistas electrónicas y las disciplinas es una circunstancia ampliamente contrastada, en la que los investigadores de ciencias resultan ser los usuarios más dedicados. Cabe señalar, no obstante, que la menor disponibilidad de suscripciones en las áreas de ciencias sociales y principalmente de las humanidades resulta un factor que juega en detrimento de su uso.

En relación al conocimiento que el PDI tiene de las revistas electrónicas, cabe apuntar que es alto y su consideración hacia ellas muy positiva. Podemos afirmar que las revistas electrónicas se han convertido en una herramienta de trabajo esencial entre el profesorado universitario. El estudio de Pinto, Fernández-Marcial y Gómez-Camarero (2010) que recoge las opiniones de investigadores de las áreas de ciencias puras, de la naturaleza y técnicas de 19 universidades españolas así lo constata.

Los investigadores que han respondido a la encuesta confirman conocer la oferta disponible y utilizar las revistas electrónicas, y consideran que éstas presentan fortalezas con respecto a las revistas en papel, entre estas ventajas señalan su facilidad de acceso y el ahorro de tiempo en la consecución de los artículos que se requieren.

El estudio de Deng (2010), basado en una encuesta que indaga la utilización de los recursos electrónicos por parte de toda la comunidad académica de una universidad australiana, muestra que las revistas electrónicas son, después de la consulta del catálogo, el recurso más empleado por los universitarios, con un porcentaje de utilización superior al $80 \%$. Este mismo autor, tras revisar la literatura sobre este particular, identifica cinco motivaciones principales para el uso de los recursos electrónicos: la facilidad de acceso, el ahorro de tiempo, la variedad de oferta, la disponibilidad de herramientas de búsqueda y la ausencia de limitaciones espaciales. En el trabajo mencionado resulta también la facilidad de acceso la razón esgrimida principalmente (86.2 \%), y el ahorro de tiempo ocupa una segunda posición con un $73.1 \%$ de las respuestas. En el trabajo de Pinto, Fernández-Marcial y Gómez-Camarero (2010) la facilidad de acceso a las revistas es el segundo ítem más valorado después de la facilidad de utilización de la web de la biblioteca y por delante de la variedad y adecuación de la oferta de revistas electrónicas, que se posiciona en el tercer lugar.

En el caso de los seis profesores que no utilizan las revistas, las razones alegadas son en tres casos el desconocimiento de la oferta de contenidos digitales disponibles. Dos investigadores señalan que se encuentran satisfechos con la colección disponible en papel en su departamento. Por último, un profesor de humanidades aduce dificultades tecnológicas y señala que la curva de aprendizaje requerida para acceder a las revistas electrónicas no lo com- 
pensa dado que la universidad no dispone de contenidos esenciales para su línea de investigación.

El conocimiento de la oferta es un factor esencial para lograr un uso efectivo, como se constata en numerosos estudios (Rehman y Ramzy, 2004). Por tanto, parece imprescindible potenciar las tareas de promoción de los contenidos disponibles por parte de las bibliotecas universitarias.

Son escasos los docentes que han realizado un curso de formación programado por la biblioteca para familiarizarse con el uso de las revistas electrónicas, si bien en el área de ciencias sociales este número es superior (7). Parecen constatarse, de un lado, las suficientes habilidades tecnológicas de los investigadores, $y$, del otro, la transparencia de los diversos sistemas de acceso empleados para acceder a las revistas electrónicas.

En cuanto a la utilidad de las revistas electrónicas, se confirma que el uso destinado a la investigación es el prioritario, e incluso se señala como fin exclusivo en muchos casos. Un significativo porcentaje de los investigadores encuestados considera que la mayor disponibilidad de contenidos electrónicos, que ha sido proporcionada por la contratación por parte de las bibliotecas universitarias de los paquetes de revistas, ha favorecido su productividad científica. Los profesores de mayor edad y superior categoría así como los más jóvenes son los menos positivos en esta cuestión, como resulta natural por haber desarrollado su carrera investigadora sin disponer de recursos electrónicos, en el primer caso, o por haberse incorporado a ella cuando ya eran accesibles, en el segundo.

El modo de acceso a los contenidos electrónicos es variado, dominan las respuestas en las que los encuestados afirman acceder a través de buscadores y por medio de la página de la biblioteca. Los investigadores de ciencias sociales muestran una decidida preferencia por la página web de la biblioteca como medio de acceso a los recursos electrónicos. Con frecuencia, en su conjunto, afirman acceder también a través de bases de datos y, con menos regularidad, directamente por medio de las páginas de los distribuidores.

Se suele hacer uso de las revistas desde el despacho de la facultad y en el horario habitual de trabajo. Casi el $100 \%$ de los encuestados señalan esta respuesta, si bien algunos profesores acceden también desde su domicilio fuera del horario laboral usual. El acceso desde el domicilio parece ser un hábito más frecuente entre los investigadores de ciencias sociales y humanidades, quizá porque las características de su investigación les permiten una menor permanencia en el centro de trabajo.

En cualquier caso, se demuestran las ventajas que tienen los recursos electrónicos con respecto a su accesibilidad independiente de su ubicación física (Hayati y Jowkar, 2008) y se constata la cada vez mayor desvinculación entre investigadores y biblioteca. 
La intensidad de uso es alta, predominan quienes afirman utilizar las revistas con frecuencia diaria o semanal. Algunos investigadores señalan una periodicidad variable en función de sus necesidades del momento. Esta respuesta es dominante entre los profesores de humanidades y muy común entre aquellos de ciencias sociales. Consideramos que la frecuencia variable guarda relación con el predominio de la búsqueda temática como mecanismo de actualización que se constata en este estudio.

Consultados acerca de si tienen periodos de mayor actividad, un porcentaje significativo de los investigadores, cercano al $40 \%$, manifiesta que no. Ésta es la respuesta más frecuente entre los docentes de CCSS y CCHH.

Entre los profesores que contestan afirmativamente, el $34 \%$ apunta que esta época se corresponde con el curso académico, circunstancia elegida por el $50 \%$ de los docentes de las áreas de ciencias sociales. Algunos profesores indican específicamente la preferencia por los periodos sin docencia dentro del año escolar.

Cuestionados sobre las revistas que consultan, la respuesta mayoritaria se corresponde con la que señala que utilizan las mismas que ya disponían en papel y otras diferentes accesibles ahora en formato digital. Se constata, así pues, la flexibilidad de la demanda a la vez que la fidelidad a las publicaciones utilizadas previamente.

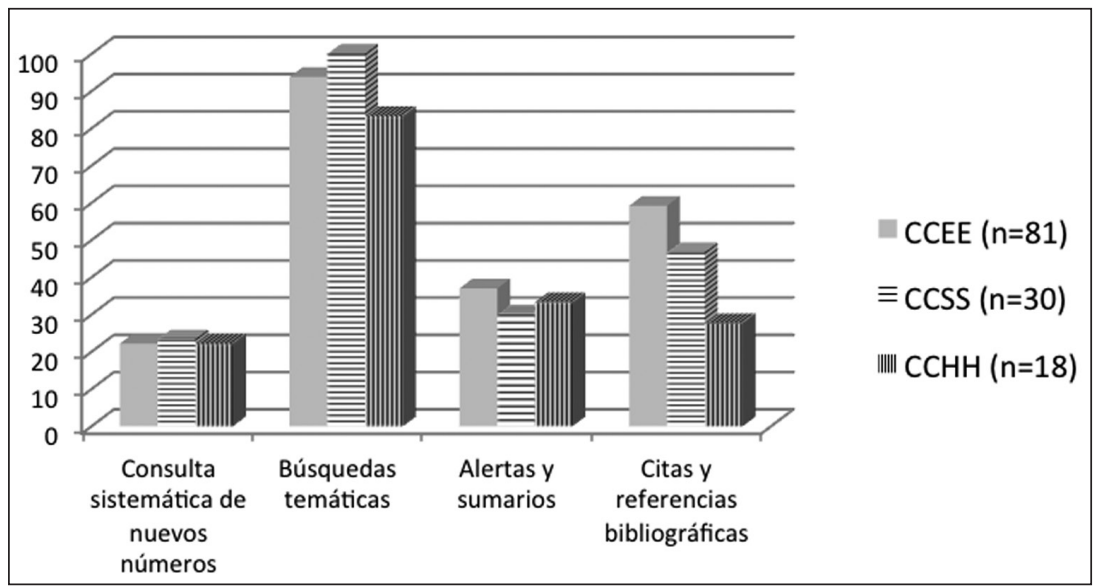

Figura 4. Estrategias para mantenerse informado

Consultados en relación a la estrategia que utilizan para mantenerse actualizados sobre los desarrollos de su campo de interés y para llevar a cabo su investigación, las respuestas son variadas, si bien son mayoría quienes afirman realizar búsquedas temáticas cuando necesitan reunir información (Figura 4); 
este resultado se complementa con el uso de alertas que no son sino búsquedas temáticas automatizadas. En la Universidad de León es frecuente también el uso de citas y referencias bibliográficas y la utilización de sumarios. El estudio de Ollé y Borrego (2010) parece constatar que el browsing ha sido sustituido, en gran medida, por las alertas recibidas por correo electrónico.

En la Universidad de León el seguimiento de los nuevos números de determinadas revistas es limitado en todos los sectores analizados, resultado que se corrobora en otra pregunta de la encuesta referida a las estrategias que utilizan los investigadores para localizar artículos, en la que las contestaciones privilegian la utilización de los formularios de búsqueda frente a los listados alfabéticos, tal y como se observa en la Figura 5. La excepción la constituyen los profesores de humanidades quienes parecen utilizar ambos mecanismos de búsqueda por igual.

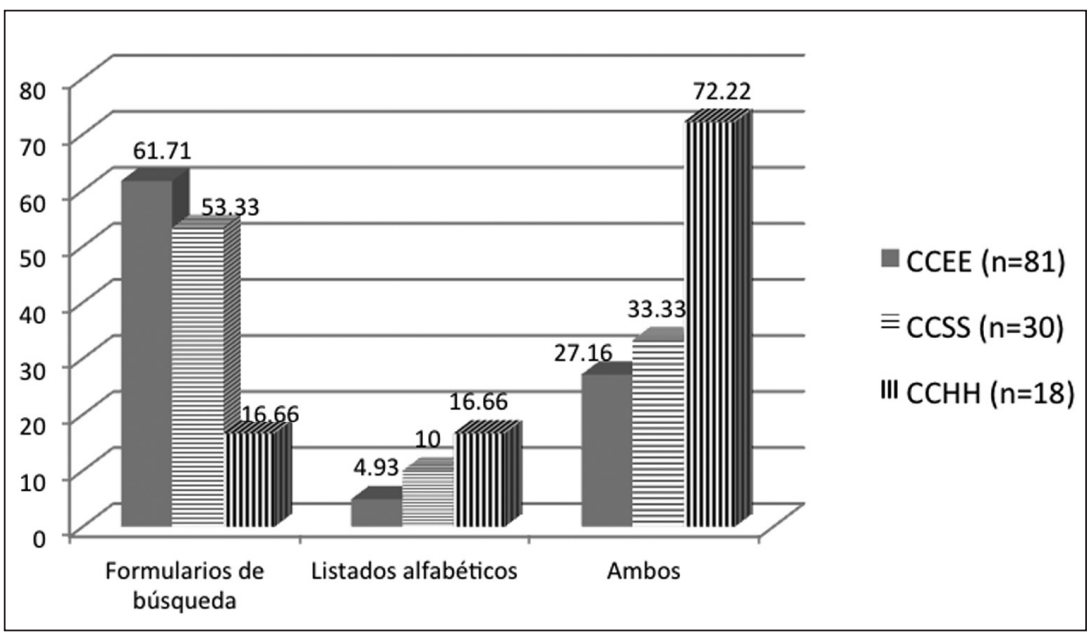

Figura 5. Estrategias para la localización de artículos

Los resultados de diferentes investigaciones fundadas en encuestas ilustran una tendencia entre los usuarios de revistas electrónicas a buscar más que a navegar (Boyce et al., 2004; Sathe, Grady y Giuse, 2002; Voorbij y Ongering, 2006; Tenopir y King, 2008; Tenopir et al., 2009). No obstante, resulta evidente que los usuarios se comportan de forma diferente en función de sus objetivos. Utilizarían la navegación para realizar su puesta al día de las novedades publicadas y la búsqueda para reunir información sobre un tema específico.

La duración de las sesiones de acceso varía entre 15 minutos y algo más de una hora, como varía también el número de artículos descargados por se- 
sión que mayoritariamente oscila entre 1 y 10 en las áreas de ciencias sociales y ciencias experimentales. Entre los profesores de humanidades resulta más frecuente la respuesta que señala que las descargas se limitan a entre 1 y 3 artículos por sesión.

En este sentido, Nicholas et al. (2008) señalan que en OhioLink el $49 \%$ de los usuarios ha visualizado una media de un artículo por sesión, algo más del $36 \%$ visualizó de 2 a 4 artículos diferentes, el $10 \%$ entre 5 y 10 y un $2 \%$ más de 21 artículos.

El mismo grupo de investigación (Nicholas et al., 2007) discute sobre los aspectos positivos y negativos que caracterizan el comportamiento de bouncing (rebote) identificado a través de exhaustivos análisis de ficheros logs en los que se evidencia que una alta proporción de usuarios ve escasos ítems o páginas durante la visita a una sede web concreta y, en muchas ocasiones, no vuelven a ese sitio o no lo hacen de modo asiduo.

En relación con los hábitos de consulta de artículos, muchos profesores indican leer en pantalla superficialmente el artículo para comprobar si es de su interés. Prácticamente todos los investigadores afirman descargar en su computadora los artículos que les interesan, y aproximadamente el $50 \%$ de los docentes de las tres áreas termina imprimiendo el documento si va a realizar una lectura más profunda.

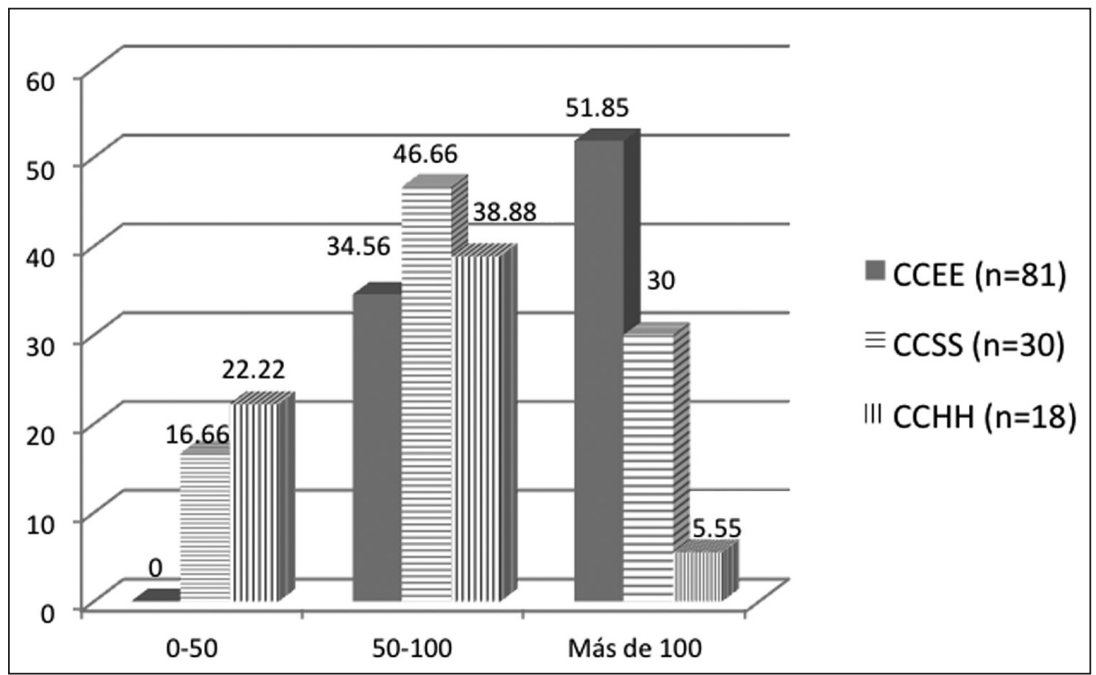

Figura 6. Consulta anual de artículos

Como se aprecia en la Figura 6, la consulta anual de artículos es elevada, todos los investigadores de ciencias experimentales afirman consultar más 
de 50 artículos aunque por lo general el número supera los 200, caso de 22 de los investigadores que respondieron a la encuesta. En el caso de los docentes de humanidades y ciencias sociales la respuesta más común se circunscribe al tramo 50-100 con algunos profesores que descargan más artículos, en el caso fundamentalmente de los académicos de ciencias sociales, y otros menos, con mayor incidencia en las áreas de humanidades.

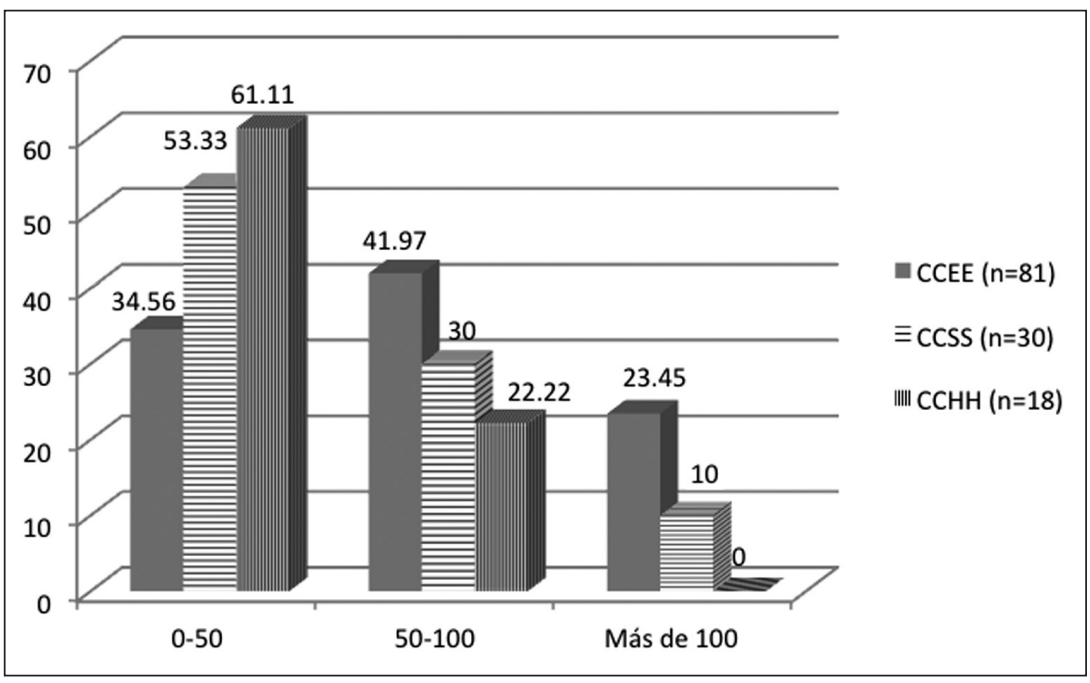

Figura 7. Descargas anuales

Esta respuesta guarda relación con el número de artículos descargados anualmente (Figura 7), que por lo general son menos que los consultados. La respuesta dominante de los profesores de ciencias experimentales se corresponde con el tramo de 50-100, la de humanidades con el tramo de 25-50, presentándose la circunstancia de que ningún profesor de $\mathrm{CCHH}$ afirma realizar más de 100 descargas anuales. Por su parte, los profesores de ciencias sociales ofrecen una respuesta muy diversificada.

Las ratios de descargas de artículos de ScienceDirect, SpringerLink y Wiley en la Universidad de León entre 2006 y 2010, como muestra la Tabla II (Apéndice 2), confirman los datos de la encuesta.

Con respecto a la lectura de artículos, la mitad de los investigadores encuestados de todas las áreas afirman leer más artículos que hace unos años. Un $25 \%$ de los investigadores de ciencias experimentales y un 30\% de los de ciencias sociales reconocen leer los artículos con menor profundidad. Asimismo, hay profesores que manifiestan no leer algunos de los artículos descargados. 
Predomina la lectura de los documentos impresos sobre la lectura en pantalla, si bien los datos se presentan bastante semejantes, de hecho en ciencias experimentales son prácticamente la mitad los investigadores quienes manifiestan leer en pantalla. Tenopir et al. (2009) apuntan que un $89 \%$ descarga en la computadora los artículos que selecciona en pantalla para leerlos posteriormente.

En relación al tiempo de lectura, Tenopir et al. (2009) indican que la media de tiempo invertido varía según el formato de la fuente consultada, donde la media es de 26 minutos para la consulta de revistas impresas y de 40 minutos para las revistas electrónicas. Nicholas et al. (2008) señalan que el tiempo empleado en la lectura en línea de un artículo completo fue de alrededor de un cuarto de hora entre el personal docente e investigador, pero que la mayoría de las veces las visitas a un texto completo son muy cortas, de tres minutos o menos, por lo que se puede hablar de lecturas superficiales.

Indagando en los factores que, aparte de la temática, tienen mayor trascendencia para la selección de un artículo, los investigadores apuntan a los objetivos y la metodología empleada en el estudio, al prestigio o impacto de la revista, y al reconocimiento de los autores. Los investigadores de humanidades valoran los resultados que se obtienen por encima de los criterios anteriores.

En el trabajo llevado a cabo por Ollé y Borrego (2010) el contenido se destaca como aspecto clave para determinar el valor y la relevancia de un artículo. La mitad de los entrevistados confían en el resumen y en el autor como principales criterios de selección. Asimismo mencionan el prestigio de la revista y la metodología empleada en el artículo como factores a considerar.

Con respecto a la antigüedad de los artículos que se seleccionan, la mayoría de los investigadores de ciencias experimentales señala que suelen consultar artículos de menos de cinco años de antigüedad. En ciencias sociales también resulta mayoritaria esta respuesta si bien un $40 \%$ manifiesta consultar también artículos de más de cinco años de antigüedad, respuesta predominante entre los profesores de humanidades. Se constata el distinto grado de obsolescencia de la investigación de las áreas analizadas. En este sentido, Tenopir et al. (2009) apuntan que cada vez se leen más artículos antiguos debido a la disponibilidad de los mismos en formato electrónico a través de backfiles.

Preguntados sobre la utilización de gestores bibliográficos, la mayoría respondió negativamente. RefWorks y EndNote Web son los gestores más utilizados. La biblioteca mantiene licencia de ambas aplicaciones y oferta cursos de formación presenciales y en línea. En este sentido, Ollé y Borrego (2010) señalan que los usuarios de las bibliotecas universitarias catalanas 
apenas utilizan gestores bibliográficos y emplean sistemas ya caducos de carpetas o bien no utilizan ningún sistema; quienes usan gestores se decantan por RefWorks por la misma razón que lo hacen los usuarios de la Universidad de León: la posesión de una licencia para su uso.

Solamente los investigadores de ciencias experimentales manifiestan un reconocimiento claro de las revistas electrónicas de los paquetes multidisciplinares de ScienceDirect, Springer y Wiley. Esta circunstancia obedece a que los tres suministradores distribuyen contenidos mayoritariamente de ciencias experimentales, ciencias de la naturaleza y de la salud.

En cuanto a la preferencia por los contenidos de estos proveedores, los investigadores encuestados de CCEE muestran su inclinación por ScienceDirect y, en segundo lugar, por las revistas de Springer (Figura 8).

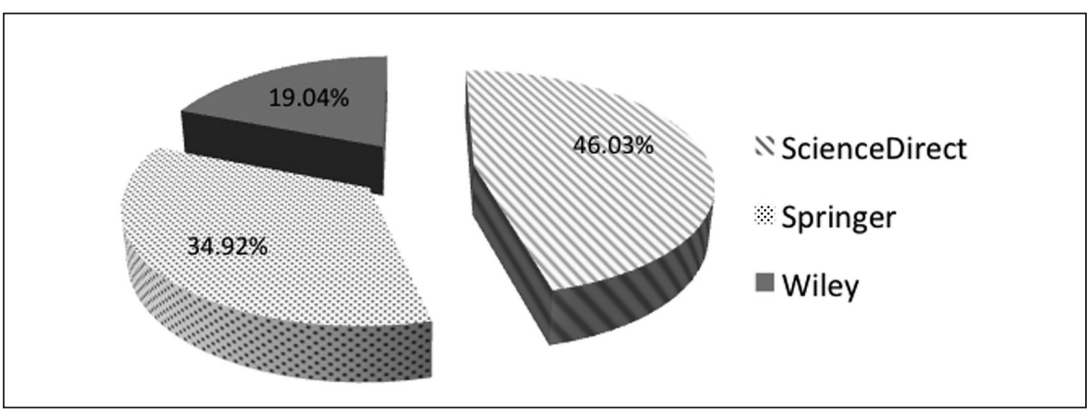

Figura 8. Preferencias de los investigadores de CCEE

Los datos de descargas de artículos de estos proveedores en la Universidad de León, como se señala en la Tabla III (Apéndice 2), confirman la preferencia de los investigadores leoneses por el paquete de Elsevier y el uso destacado que hacen de los contenidos de Springer con respecto a los de Wiley en la mayoría de los años estudiados.

Los investigadores de CCSS que responden a esta pregunta manifiestan una preferencia por ScienceDirect y Emerald, paquetes especializados en ciencias sociales y en tercer lugar señalan los contenidos de Springer.

Los investigadores de humanidades no muestran una clara inclinación por ninguno de los paquetes mencionados, siendo, no obstante, el de Wiley el que recaba mayor aquiescencia con 4 respuestas. Otros cuatro investigadores señalan la utilización de Dialnet y JSTOR.

La encuesta finalmente indagaba sobre la cantidad de títulos usados prioritariamente para solicitar a los investigadores en las siguientes cuestiones que explicitasen sus títulos preferidos para desarrollar su investigación y para publicar sus resultados. Los profesores leoneses presentan una respuesta 
bastante diversificada, que oscila entre el seguimiento de menos de 5 títulos y más de 20 (Figura 9). En las tres áreas predominan las respuestas que señalan la consulta sistemática a menos de diez revistas.

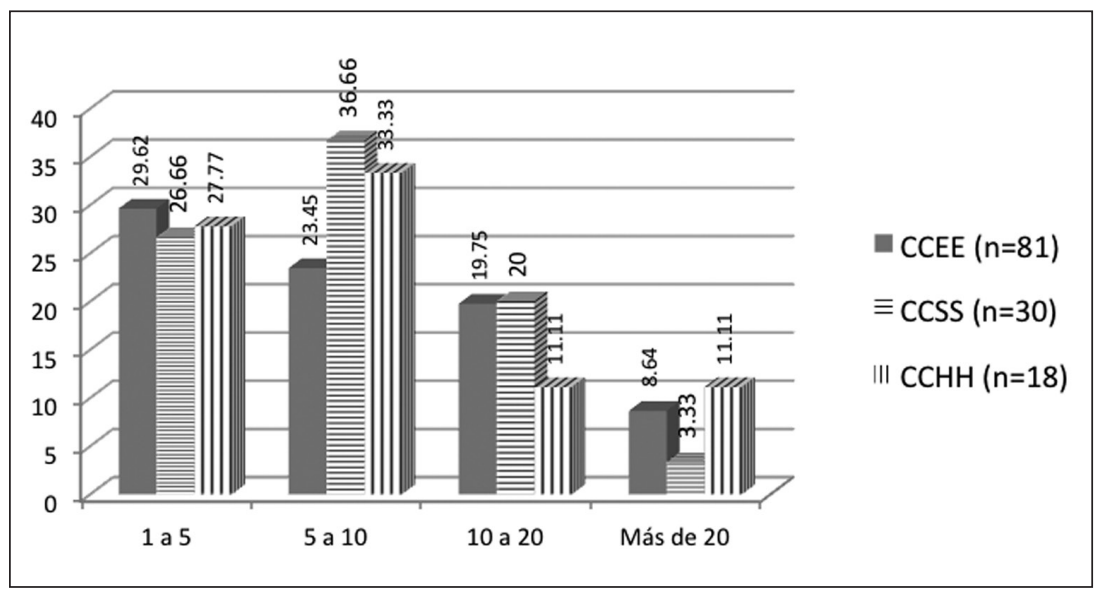

Figura 9. Número de títulos de uso prioritario

En estudios basados en estadísticas de uso de revistas se ha constatado que el grueso de las descargas se realiza a partir de un porcentaje reducido de publicaciones. Esta considerable concentración de uso se muestra coherente con los resultados hallados en investigaciones previas de Nicholas y su grupo de investigación CIBER, que confirman el hábito de un importante número de investigadores a concentrar su uso en un número limitado de títulos, si bien en el trabajo aquí presentado se supera el umbral de 3 revistas hallado por Nicholas y Huntington (2006).

\section{Análisis de los títulos de uso prioritario y comparación con el Top 25 de descargas y de visibilidad}

El número de respuestas recabadas de las preguntas 23 y 24 , en las que se solicitaba a los investigadores que apuntasen los títulos que más leían y los títulos en que más publicaban, son más limitadas. En el primer caso contestan 98 investigadores; a la segunda pregunta responden 83 profesores.

Se incluyen en la Tabla IV (Apéndice 2) los títulos por los que han mostrado preferencia en la encuesta más de dos investigadores. Se trata de un total de 29 revistas: 22 son títulos de ciencias experimentales, de la naturaleza y de la salud, y 7 son títulos de humanidades y ciencias sociales. 
Con respecto a los principales títulos usados para el desarrollo de la investigación de los científicos leoneses, se aprecia una extraordinaria variedad y dispersión. Son escasas las revistas utilizadas prioritariamente por más de dos investigadores. Las coincidencias, si bien mínimas, son más comunes entre profesores pertenecientes a la misma área de conocimiento, y se constatan preferencias más homogéneas en el departamento de Higiene y Tecnología de los Alimentos. En las áreas de ciencias sociales y humanidades el grado mayor de acuerdo se observa en Biblioteconomía y Documentación, Historia del Arte y Psicología.

La correspondencia entre títulos consultados y revistas elegidas para comunicar la investigación es limitada, circunstancia que consideramos tiene su origen en el hecho de que, en muchas ocasiones, los académicos leen revistas más reputadas que aquellas en que publican posteriormente su investigación (Tabla IV, Apéndice 2). No obstante, sí se aprecia cierta coherencia en las respuestas de los investigadores entre las revistas que leen y aquellas que utilizan para publicar, fundamentalmente en el caso del departamento de Higiene y Tecnología de los Alimentos.

En la Tabla IV (Apéndice 2) se observa la escasa coincidencia mencionada de ambas prioridades que se circunscribe a 7 títulos, lo que supone un $25 \%$ de los títulos que se consideran prioritarios para más de dos investigadores. Se trata de las revistas siguientes: El Profesional de la Información, Food Microbiology, Forest Ecology and Management, International Journal of Food Microbiology, Journal of Applied Microbiology, Journal of Food Protection y Plant Ecology. De estos títulos, cinco están disponibles en formato electrónico en los suministradores de contenidos suscritos por la Universidad de León. En concreto, tres son distribuidos por ScienceDirect, uno por Springer y otro por Wiley. A ellos se suma la revista El Profesional de la Información, que dispone de versión electrónica, y Journal of Food Protection, editada por la International Association for Food Protection (IAFP).

Con respecto a la coincidencia de los títulos preferentes de consulta extraídos de las encuestas y los títulos seleccionados por el número de descargas acumulativas 2006-2010 (Top 25) de los suministradores pluridisciplinares ScienceDirect, SpringerLink y Wiley, cabe señalar que son 6 las revistas coincidentes. Se trata de Applied and Environmental Microbiology, Food Microbiology, Forest Ecology and Management, International Journal of Food Microbiology, Journal of Food Protection y Meat Science. En concreto, la revista International Journal of Food Microbiology ocupa la primera posición con 7655 descargas y Meat Science la tercera con 6 014. Por su parte, Theriogenology ocupa la segunda posición con 6671 descargas acumulativas y aparece mencionada en la encuesta como revista preferida para la comunicación cien- 
tífica. Los tres títulos están distribuidos por ScienceDirect. Como puede observarse se trata de revistas mayoritariamente de ciencias de la alimentación, a las que se suma un título de microbiología y otro de ecología.

En relación con la correspondencia entre los títulos preferentes para difundir la investigación y las revistas seleccionadas de mayor visibilidad en las bases de datos WoS y Scopus entre 2006 y 2011 (Top 25), la coincidencia es de 9 publicaciones, 6 de las cuales son también las más leídas que citamos arriba. A estos títulos cabe añadir Atmospheric Research, Journal of Pineal Research y Theriogenology. Se suman a las revistas preferidas dos títulos de ciencias de la salud y otra publicación de ecología. Theriogenology y Atmospheric Research ocupan la segunda y la quinta posición, respectivamente, en el número de artículos publicados por investigadores de la Universidad de León.

Las revistas identificadas muestran un predominio de temáticas relacionadas con las ciencias de la alimentación, botánica, ecología, medio ambiente, microbiología y producción vegetal en consonancia, en gran medida, con el más elevado número de respuestas procedentes del departamento de Biodiversidad y Gestión Ambiental, en concreto del área de conocimiento de Botánica, y el mayor grado de aquiescencia entre los científicos del departamento de Higiene y Tecnología de los Alimentos.

En los estudios que hemos realizado a partir de las estadísticas de uso proporcionadas por los proveedores, se observa un perfil de consumo con un sesgo marcado por el peso de los estudios de veterinaria en León. Esta situación no se aprecia en este trabajo debido a la escasez de respuestas por parte de los investigadores de algunas de las áreas de la Facultad de Veterinaria, como es el caso de Producción Animal y Sanidad Animal, en las que se ha constatado el más elevado nivel de productividad en $W o S$ en la Universidad de León, posicionándose por encima de áreas como Microbiología, Ecología y Medicina y Cirugía Animal, que conforman el top 5 de productividad científica en esta institución.

Con la excepción de dos revistas españolas del ámbito de las humanidades, el Anuario del Departamento de Historia y Teoría del Arte e Hispania: Revista Española de Historia, los restantes títulos seleccionados se hallan recogidos en las bases de datos Web of Science y disponen de Factor de Impacto en el JCR. En el caso de Scopus se observan las mismas ausencias, a las que se suma la de la revista Journal of Biological Chemistry.

Finalmente queremos mencionar las sugerencias recogidas en el apartado que se facilitaba al final de la encuesta. Los investigadores que lo utilizaron, un total de 10 entre los que figura un profesor de humanidades y los restantes de ciencias experimentales, solicitan mayoritariamente la suscripción a más revistas de su ámbito. Se hace mención en varias ocasiones a las revistas de Nature. 
Un investigador del Área de Bioquímica y Biología Molecular, pertenenciente a la categoría Contratado Doctor, realiza la siguiente reflexión: "Necesitaríamos mejores revistas en calidad, como Nature. No hace falta tener cientos de revistas porque muchas de ellas tienen el mismo valor científico que la hoja parroquial de una localidad rural".

Asimismo, son dos los investigadores que hacen referencia a la necesidad de renovar el equipamiento tecnológico ya que resulta difícil el acceso y la descarga de artículos porque las interfaces son poco intuitivas.

\section{Conclusiones}

El estudio presentado nos ha permitido aproximarnos al comportamiento, las preferencias y el nivel de satisfacción de la comunidad académica de la Universidad de León con respecto a las revistas electrónicas. El análisis de los resultados de la encuesta, a la que dieron respuesta 135 investigadores de esta institución, creemos que revela un notable grado de aceptación y conocimiento de los contenidos electrónicos contratados; solamente seis de los docentes que respondieron a la encuesta manifestaron no utilizar las revistas científicas electrónicas. Estos resultados se corresponden con la línea mostrada en el trabajo de Borrego (2005) en relación a las universidades catalanas, donde un $95 \%$ de los encuestados señalaba conocer las revistas electrónicas.

Como pone de relieve Deng (2010) el conocimiento de la oferta y la calidad de los contenidos electrónicos disponibles son los dos factores principales para hacer un uso efectivo y eficiente de los recursos electrónicos. Por su parte, la incapacidad para explotar el potencial al alcance se debe atribuir a la falta de conocimiento de los recursos disponibles, a la ausencia de competencia tecnológica, a la falta de entrenamiento y/o a la carencia de tiempo suficiente para utilizar la oferta de recursos electrónicos.

Atendiendo al área de conocimiento y a los datos demográficos expresados, podemos identificar como usuarios mayoritarios de e-revistas a profesores titulares de entre 41 y 60 años pertenecientes al ámbito de ciencias experimentales. Cabe indicar que el $50 \%$ de las respuestas proceden de este campo, predominantemente de las especialidades de Botánica, Ecología y Microbiología. No se han apreciado disparidades en los hábitos de uso entre hombres y mujeres ni se ha detectado rechazo al formato electrónico por parte de los investigadores de mayor edad.

Se observa un empleo más intenso de las revistas electrónicas por parte de los investigadores de ciencias experimentales, de la naturaleza y de la salud. 
Asimismo, se manifiesta mayor grado de uso por parte de los docentes del campo de las ciencias sociales respecto a aquellos de humanidades.

Estudios realizados con anterioridad, fundados en las estadísticas de uso de varias universidades del noroeste español (Rodríguez y Alvite, 2006a, 2006b; Rodríguez et al., 2008; Rodríguez y Alvite, 2011; Rodríguez, Alvite y Barrionuevo, 2012), han permitido comprobar que son los científicos de las áreas de ciencias experimentales y de la salud los usuarios más dedicados, a juzgar por el volumen de descargas de artículos y el número de publicaciones de estos campos temáticos.

Los resultados hallados en el presente estudio confirman que el empleo prioritario de las revistas electrónicas se orienta a tareas relacionadas con la investigación, tendencia ya señalada en buena parte de la literatura. Sirven de ejemplo los trabajos de Tenopir y King (2000), quienes señalan un porcentaje del $75 \%$ de usuarios que se decantan por el uso de e-revistas con un finalidad investigadora, frente al $41 \%$ que apuntan a fines docentes. Dilek-Kayaoglu (2008) sitúa esa prioridad investigadora en un $67.5 \%$.

Nuestro estudio revela una frecuencia de uso intensa, en la que predominan las respuestas que señalan un empleo diario o semanal de revistas electrónicas. El estudio de Deng (2010) confirma esta tendencia, ya que más del $59 \%$ de las respuestas a la encuesta efectuada señalan una frecuencia de utilización superior a una vez a la semana.

Los investigadores encuestados confirman, de un lado, la elasticidad de uso, consustancial a la oferta de paquetes de revistas electrónicas; del otro, la concentración del empleo masivo en un limitado número de títulos. Asimismo, se observa fidelidad a títulos reputados que ya se venían usando en formato impreso.

En cuanto al modo de acceso, hemos de resaltar la heterogeneidad de respuestas aportadas. Los investigadores de ciencias sociales manifestaron una clara predilección por el acceso a través del portal de la biblioteca, si bien en el resto de las áreas predominaron las respuestas que indican el uso preferente de los motores web para el acceso a las revistas electrónicas y una utilización poco frecuente de las plataformas de los distribuidores. Esta inclinación por los motores web se constata igualmente en un buen conjunto de trabajos; así, Ollé y Borrego (2010) corroboran un mayoritario acceso a través de Google debido a la mayor facilidad de uso del motor frente a la web de la biblioteca. Por su parte, Rowlands (2007) confirma que el profesorado hace un escaso uso de la página web de la biblioteca. Creemos que la tendencia observada llama la atención sobre una cierta desafección hacia la biblioteca por parte de la comunidad académica.

Nicholas (2008) alerta sobre el éxodo de los usuarios desde las bibliotecas hacia los editores, que se acelerará con el desarrollo de la oferta bibliotecaria de e-books al sumarse al proceso migratorio los estudiantes y los investigadores 
de las áreas de ciencias sociales y humanidades, menos proclives al uso de las revistas.

Resulta elocuente, en este mismo sentido, el escaso número de profesores que responden a nuestra encuesta manifestando haber seguido cursos de formación para optimizar el uso de las revistas electrónicas. Parece que es una tendencia extrapolable al ámbito de la investigación en España; así, Pinto, Fernández-Marcial y Gómez-Camarero (2010) constatan que los académicos se muestran reticentes con respecto a la adopción, por parte de los bibliotecarios, de un papel más activo en la mediación de información y conceden poco valor al rol que el bibliotecario puede jugar en cuanto a su formación en competencias informativas. Tampoco los investigadores catalanes recurren a los bibliotecarios para filtrar el exceso de información que reciben (Ollé y Borrego, 2010). Como señala Chiu (2000), los investigadores parecen confundir el conocimiento de su campo de dedicación con la habilidad para encontrar información útil sobre un tema determinado.

Convenimos con las sugerencias del informe reciente de RLUK (Auckland, 2012), en el que se plantean nuevas estrategias para dar respuesta, por parte de la biblioteca, a las necesidades de los investigadores, haciendo hincapié en la necesidad acuciante de contar con bibliotecarios especializados por materias. Se trata de que la biblioteca entienda a la comunidad investigadora como un grupo heterogéneo, con necesidades de información que difieren atendiendo a la disciplina en la que trabajan, la cultura y praxis investigadora, y el estadio de la carrera en el que se encuentran. Los investigadores demandan servicios personalizados adaptados a sus particulares flujos de trabajo.

Consideramos que la respuesta de la biblioteca en los últimos años se ha basado más en el desarrollo tecnológico y en la integración de los contenidos que se le ofertaban desde las editoriales que en un conocimiento riguroso de los usuarios a los que dirige sus servicios.

Los resultados de la Universidad de León inciden en mostrar una clara preferencia por el empleo de estrategias de búsqueda para el acceso a la información frente a las técnicas de navegación; además, las búsquedas temáticas resultan la opción mayoritariamente elegida por los investigadores de esta universidad para mantenerse informados. Dilek-Kayaoglu (2008) señala un porcentaje del $80.3 \%$ de usuarios que muestran una clara inclinación hacia la búsqueda por materias frente a la navegación por listas de títulos. Nicholas, Huntington y Jamali (2007) matizan en su trabajo ciertos aspectos sobre las técnicas de búsqueda, indicando que el $52 \%$ se corresponde con búsquedas simples, $41 \%$ con búsquedas avanzadas y un $7 \%$ emplea ambas. Consideramos igualmente relevante la distinción observada por estos mismos autores 
en OhioLINK que señala una predilección por los mecanismos de navegación en las universidades de investigación extensiva y una preferencia por las utilidades de búsqueda en las universidades orientadas a la docencia.

La media de artículos anuales consultados recogida en la encuesta es considerable, con diferencias manifiestas entre las áreas de humanidades, ciencias sociales y ciencias experimentales, siendo en esta última donde el volumen supera en muchos casos los 200 artículos. El número de títulos de revistas consultado de modo regular se sitúa en una media inferior a 10. En cuanto a la descarga anual de ítems, la encuesta confirma estudios estadísticos previos en los que la ratio por investigador en el año 2010 se fijó en 111.47.

Las respuestas de la encuesta manifiestan que la mitad de los profesores leen más artículos que años atrás; además, entre un $25 \%$ y un $30 \%$ hablan de una lectura con menor profundidad. En este sentido, el estudio de Rowlands (2007) indica que el profesorado lee una media de 384 documentos al año, de los que 161 son artículos de revista. Por su parte, el trabajo de Wang (2010), centrado en el comportamiento de los docentes del área de ciencias sociales en la National Cheng-chi University de Taiwán, alerta sobre las importantes diferencias en los hábitos de uso localizados en esta comunidad académica con respecto a los establecidos en los trabajos de Tenopir et al. (2005 y 2009), centrados en el área de ciencias experimentales. Las ratios señaladas por Wang se fijan en 195 artículos leídos anualmente por investigador y una dedicación media de lectura de 135 minutos por artículo.

Los investigadores del área de ciencias experimentales de la Universidad de León muestran que identifican claramente los paquetes de revistas electrónicas de los proveedores multidisciplinares contratados por la biblioteca. Las preferencias manifestadas concuerdan con los datos de descargas ya observados en trabajos previos (Rodríguez, Alvite y Barrionuevo, 2012). El paquete de ScienceDirect es el favorito, seguido de Springer en el campo de CCEE y Emerald en el de CCSS.

En cuanto a los títulos de revista de uso prioritario, puestos en correlación los títulos que los propios investigadores especifican en la encuesta con aquellos procedentes de las estadísticas de descargas y los recogidos en las bases de datos de productividad científica, se evidencia una gran disparidad que consideramos guarda relación con la especialización de la investigación del personal docente y con la dispersión en el consumo de información propiciada por las propias características de las plataformas de contenidos electrónicos.

Trataremos de profundizar en los hábitos de consumo de las distintas áreas científicas, conocimiento esencial para que las bibliotecas y los grandes grupos editores puedan ofrecer servicios y productos más adaptados que 
permitan incrementar el nivel de satisfacción de los usuarios. En este sentido, las bibliotecas universitarias deberán perseguir el equilibrio en la contratación de contenidos temáticos para poder satisfacer a los investigadores de las áreas peor cubiertas, como las humanidades.

Consideramos conveniente indagar la desvinculación observada entre los investigadores y la biblioteca en cuanto al acceso concreto a las revistas electrónicas, en tanto interesa ahondar en los posibles cambios de percepción de la biblioteca derivados de la incorporación de herramientas tecnológicas de búsqueda única -plataformas de descubrimiento o de búsqueda federadaasí como en los valores añadidos que pudiesen aportar bibliotecarios especializados o cursos de formación realmente adaptados a las áreas de conocimiento de la comunidad académica.

Asimismo, seguiremos analizando la interrelación existente entre la utilización de las revistas electrónicas y la calidad docente e investigadora. Si bien las compras consorciadas de paquetes han sido un éxito a juzgar por los datos de utilización y de satisfacción de los usuarios, especialmente en universidades pequeñas y medianas como las que nos ocupan, nos interesa profundizar en el retorno de la inversión en productividad que el incremento de las adquisiciones y el tiempo ahorrado por la facilidad de acceso a los recursos electrónicos supone.

\section{Agradecimientos}

Este estudio muestra los resultados del proyecto de investigación financiado por la Junta de Castilla y León (BOCyL 29 de marzo de 2011) "Rentabilidad de la inversión en revistas electrónicas en las bibliotecas universitarias de Castilla y León (LE013A11-2)”. Investigador responsable: Dra. Blanca Rodríguez Bravo.

Agradecemos la colaboración de los alumnos Ana Belén Álvarez García, María Antonia Fernández Rodríguez, Laura González Casares, Concepción Méndez Fernández y Ángel Vidal Madrid en la elaboración de la encuesta y en la gestión de su envío y recepción. 


\section{BiBLIOGRAFÍA}

Armstrong, C. et al. (2001), "A study of the use of electronic Information systems by higher education students in the UK", en Program: electronic library and information systems, vol. 35, núm. 3, pp. 241262.

Aukland, M. (2012), Re-Skilling for Research: Investigating the Needs of researchers and How Library Staff Can Best Support Them, London: RLUK-research Libraries United Kingdom. Disponible en: http://www.rluk.ac.uk/files/RLUK\%20Re-skilling.pdf

Borrego, A. (2005) (dir.), Estudi d'usuaris de les revistes electròniques del CBUC, Consorci de Biblioteques Universitàries de Catalunya. Disponible en: http://www.recercat.net/bitstream/2072/1439/1/ ESTUDI+USUARIS.pdf

- et al. (2007), "Use and users of electronic journals at Catalan universities: the results of a survey", en Journal of Academic Librarianship, vol. 33, núm. 1, pp. 67-75.

_- Urbano, C. (2007), "Analysis of the behaviour of the users of a package of electronic journals in the field of chemistry", en Journal of Documentation, vol. 63, núm. 2, pp. 243-258.

Boukacem-Zeghmouri, C.; Schöpfel, J. (2008), "On the usage of e-journals in French universities", en Serials: The Journal for the Serials Community, vol. 21, núm. 2, pp. 121-126.

Boyce et al. (2004), "How electronic journals are changing patterns of use”, en The Serials Librarian, vol. 46, núm. 1/2, pp. 121-141.

Brady, E. E.; McCord, S. K.; Galbraith, B. (2006), "Print versus Electronic Journal Use in Three Sci/Tech Disciplines: The Cultural Shift in Process", en College E Research Libraries, vol. 67, núm. 4, pp. 354-363. Disponible en: http://crl.acrl.org/content/67/4/354. full.pdf

Chiu, Ha-lin (2000), "Seeking help in Hong Kong's academic libraries”, en OCLC Systems E Services, vol. 16, núm. 4, pp. 181-188.

CIBER (Centre for Information Behaviour and the Evaluation of Research) (2011), E-Journals: Their Use, Value and Impact: Final Report, London: City University. Disponible en: http://www.rin. ac.uk/news/e-journals-their-use-value-and-impact-final-reportf

Delgado López-Cozar, E.; Ruiz-Pérez, R.; Jiménez-Contreras, E. (2006), La edición de revistas científicas. Directrices, criterios y modelos de evaluación, Madrid: FECyT. Disponible en: http://www. fecyt.es/fecyt/detalle.do?elegidaNivel1=;SalaPrensa\&elegidaNivel 2=;SalaPrensa;publicaciones\&elegidaNivel $3=$;SalaPrensa;publica ciones; guiasymanuales $\&$ tc $=$ publicaciones $\&$ id $=$ Edicion + revistas + cientificas

Deng, H. (2010), "Emerging patterns and trends in utility electronic resources in a higher education environment: an empirical analysis”, en New Library World, vol. 111, núm. 3/4, pp. 87-103. 
Dilek-Kayaoglu, H. (2008), "Use of Electronic Journals by Faculty at Istanbul University, Turkey: The Results of a Survey", en The Journal of Academic Librarianship, vol. 34, núm. 3, pp. 239-247.

Gargiulo, P. (2003), "Electronic journals and users: the CIBER experience in Italy”, en Serials, vol. 6, núm. 3, pp. 293-298.

Hayati, Z.; Jowkar, T. (2008), "Adoption of electronic reference materials in academic libraries of Iran", en The International Information EL Library Review, vol. 40, pp. 52-63.

Huntington, P. et al. (2006), "Article decay in the digital environment: an analysis of usage of OhioLINK by date of publication, employing deep log methods", en Journal of the American Society for Information Science and Technology, vol. 57, núm. 13, pp. 1840 1851.

Massad, V. J.; Brown, M. L.; Tucker, J. M. (2011), “The acceptance of electronic journals by business faculty: an adoption/diffusion approach", en Journal of Behavioral Studies in Business, vol. 3. Disponible en: http://www.aabri.com/manuscripts/10729.pdf

Nicholas, D. (2008), "If we do not understand our users, we will certainly fail", en The E-Resources Management Handbook 1, United Kingdom Serials Group. Disponible en: http://uksg.metapress. com/content/e2qjfqymrrmg3rqd/fulltext.pdf

_ et al. (2006), "What deep log analysis tells us about the impact of big deals: case study OhioLINK", en Journal of Documentation, vol. 62, núm. 4, pp. 482-508.

_ et al. (2007), "Characterising and evaluating information seeking behaviour in a digital environment: Spotlight on the 'bouncer”, en Information Processing \& Management, vol. 43, núm. 4, pp. 1085-1102.

et al. (2008), "Viewing and Reading behaviour in a virtual environment: the full-text download and can be read into it", en Aslib Proceedings: New Information Perspectives, vol. 60, núm. 3, pp. 185-198.

; Huntington, P. (2006), "Electronic journals: are they used?", en Interlending \& Document Supply, vol. 34, núm. 2, pp. 48-50.

—; Huntington, P.; Jamali, H. R. (2007), "Diversity in the information seeking behaviour of the virtual scholar: institutional comparisons", en Journal of Academic Librarianship, vol. 33, núm. 6, pp. 629-638.

Ollé, C.; Borrego, A. (2010), "A qualitative study of the impact of electronic journals on scholarly information behavior", en Library EInformation Science Research, vol. 32, núm. 3, pp. 221-228.

Pinto, M.; Fernández-Marcial, V.; Gómez-Camarero, C. (2010), “The impact of Information Behaviour in Academic Library Service Quality: a case study of the Science and Technology Area in Spain”, en The Journal of Academic Librarianship, vol. 36, núm. 1, pp. 70-78. 
Rehman, S.; Ramzy, V. (2004), "Internet use by health professionals at the Health Sciences Centre of Kuwait University", en Online Information Review, vol. 28, núm. 1, pp. 53-60.

Rodríguez Bravo, B. et al. (2008), "Patterns of Use of Electronic Journals in Spanish University Libraries”, en Serials Review, vol. 34, núm. 2, pp. 115-128.

; Alvite Díez, M. L. (2006a), "The Use of Electronic Journals in Academic Libraries in Castilla and León (Spain)”, en P. Brophy, J. Craven, M. Markland (eds.), Libraries Without Walls 6: Evaluating the Distributed Delivery of Library Services, London: Facet Publishing, pp. 125-137.

; Alvite Díez, M. L. (2006b), "Uso de las revistas-e suministradas por Emerald en bibliotecas universitarias españolas (2002-2005)”, en El Profesional de la Información, vol. 15, núm. 6, pp. 464-472. -; Alvite Díez, M. L. (2011), "An Analysis of the Use of Electronic Journals in a Spanish Academic Context: Developments and Profitability", en Serials Review, vol. 37, núm. 3, pp. 181-195.

; Alvite Díez, M. L.; Barrionuevo Almuzara, L. (2012), "Trends and Models in the Consumption of Electronic Contents. An Analysis of the Journals Most Widely Used in Spanish Universities", en Journal of Academic Librarianship, vol. 38, núm. 1, pp. 42-59.

Rowlands, I. (2007), "Electronic journals and user behavior: a review of recent research", en Library \& Information Science Research, vol. 29, núm. 3, pp. 369-396.

Rusch-Feja, D.; Siebeky, U. (1999), "Evaluation of usage and acceptance of electronic journals", en D-Lib Magazine, vol. 5, núm. 10. Disponible en: http://www.dlib.org/dlib/october99/rusch-feja/10rusch-feja-summary.html

Sathe, N. A.; Grady, J. L.; Giuse, N. B. (2002), "Print versus electronic journals: a preliminary investigation into the effect of journal format on research processes", en Journal of the Medical Library Association, vol. 90, núm. 2, pp. 235-243.

Tenopir, C. (2002), "Online serials heat up”, en Library Journal, vol. 127, pp. 37-38.

(2003), Use and users of electronic library resources: an overview and analysis of recent research studies, Washington, D. C.: Council on Library and Information Resources. Disponible en: http:// www.clir.org/pubs/reports/pub120/pub120.pdf

et al. (2005), "Relying on Electronic Journals: Reading Patterns of Astronomers", en Journal of the American Society for Information Science and Technology, vol. 56, núm. 8, pp. 786-802.

et al. (2009), "Electronic Journals and changes in scholarly articles seeking and reading patterns", en Aslib Proceedings: New Information Perspectives, vol. 61, núm. 1, pp. 5-31. 
Tenopir, C.; King, D. W. (2000), Towards Electronic Journals: Realities for Scientists, Librarians and Publishers, Washington: Special Libraries Association.

—; King, D. W. (2002), "Reading behaviour and electronic journals”, en Learned Publishing, vol. 15, pp. 259-265.

_ ; King, D. W. (2008), "Electronic Journals and changes in scholarly article seeking and reading patterns", en D-Lib Magazine, vol. 14, núm. 11/12. Disponible en: http://www.dlib.org/dlib/november08/tenopir/11tenopir.html

Town, S. (2004), "E-measures: a comprehensive waste of time?", en Vine, vol. 34, núm. 4, pp. 190-195.

Voorbij, H.; Ongering, H. (2006), “The use of electronic journals by Dutch researchers: a descriptive and exploratory study", en The Journal of Academic Librarianship, vol. 32, núm. 3, pp. 223-237.

Wang, M. (2010), "Scholarly journal use and reading behavior of social scientists in Taiwan", en The International Information E Library Review, vol. 42, núm. 4, pp. 1057-2317. 


\section{Apéndice 1}

\section{ENCUESTA}

Facultad:

Departamento:

Área de conocimiento:

Categoría profesional:

Edad
$\square 21-30$
$41-50$
$61-70$
$\square 31-40$
$\square 51-60$

Sexo
$\square$ Hombre
Mujer

Por favor señale con una cruz las afirmaciones con las que se encuentre de acuerdo.

1. Conocimiento de la oferta de revistas electrónicas accesible en la Universidad de León (Si no conoce o no utiliza las revistas electrónicas pase a la pregunta 25).

$\square$ Conozco la oferta de revistas electrónicas existente.

$\square$ Utilizo las revistas electrónicas de la universidad.

$\square$ Resulta sencillo acceder a las revistas electrónicas.

$\square$ He realizado algún curso de formación de la biblioteca para familiarizarme con el acceso a las revistas electrónicas.

$\square$ Considero que las revistas electrónicas presentan ventajas con relación a las revistas en papel.

2. Razones de la utilización de las revistas electrónicas.

$\square$ Su fácil acceso.

$\square$ El ahorro de tiempo.

$\square$ La abundancia de títulos.

$\square$ Otros:

3. Finalidad de la utilización de las revistas electrónicas.
$\square$ Docencia
$\square$ Investigación
$\square$ Otros:

4. La mayor disponibilidad de contenidos de los últimos años ha favorecido mi productividad científica. He publicado más.
$\square$ Sí
$\square$ No
$\square$ Otros: 
5. Modos de acceso a los contenidos electrónicos.

$\square$ A través de la página de la biblioteca.

$\square$ Por medio de Google u otros buscadores.

$\square$ A través de bases de datos.

$\square$ Por medio de los distribuidores de revistas electrónicas.

$\square$ Otros:

6. Lugar y periodo preferente de acceso a las revistas electrónicas.

$\square$ Desde mi despacho del departamento en mi horario habitual de trabajo en la facultad.

$\square$ Desde mi domicilio a otras horas durante los días laborables.

$\square$ Desde mi domicilio los fines de semana.

$\square$ Otros:

7. Frecuencia de acceso a las revistas electrónicas.

$\square$ Diariamente. $\quad \square$ Trimestralmente.

$\square$ Semanalmente. $\quad \square$ Una o dos veces al año.

$\square$ Quincenalmente. $\quad \square$ Periodicidad variable.

$\square$ Mensualmente.

8. Época al año de mayor actividad.

$\square$ Ninguna.

$\square$ Durante el curso académico.

$\square$ En los periodos sin docencia durante el curso académico.

$\square$ En periodos vacacionales.

$\square$ Otros:

9. Consulta de revistas específicas.

$\square$ Las mismas de que disponía en papel.

$\square$ Revistas diferentes en formato digital.

$\square$ Las mismas de que disponía en papel y otras diferentes en formato digital.

$\square$ Otros:

10. Estrategias empleadas para mantenerse al día en su campo de conocimiento y para desarrollar su investigación.

$\square$ Consulto sistemáticamente los números nuevos de las mismas revistas.

$\square$ Realizo búsquedas temáticas cuando necesito información sobre un tema concreto.

$\square$ Utilizo alertas y sumarios.

$\square$ Utilizo citas y referencias bibliográficas.

$\square$ Por medio de listas de distribución y/o contacto con compañeros.

$\square$ Otros:

11. Hábitos de consulta de artículos.

$\square$ Leo en pantalla el resumen u otras partes del documento de manera superficial para 
comprobar que me interesa el artículo.

$\square$ Guardo en mi ordenador los artículos que me interesan.

$\square$ Imprimo los artículos que me interesan.

$\square$ Algunos de los artículos descargados no llego a leerlos nunca.

12. Número de artículos electrónicos consultados anualmente (en pantalla o impresos).
$\square 1-25$
$\square$ 100-200
$\square 25-50$
$\square$ Más
$\square 50-100$

13. Número de artículos descargados anualmente (guardados y/o impresos).
$\square 1-10$
$\square$ 10-25
$\square$ 50-100
$\square 25-50$
$\square$ Más de 100

14. Duración de las sesiones de acceso.

$\square 15$ minutos.

$\square$ Media hora.

$\square$ Una hora.

$\square$ Más de una hora.

15. Número de artículos descargados por sesión.
$\square 1-3$
$\square$ 4-10
$\square$ Más de 10

16. Estrategias empleadas para la localización de artículos.
$\square$ Utilizo los formularios de búsqueda.
$\square$ Utilizo los listados alfabéticos.
$\square$ Ambos indistintamente.
$\square$ Otros:

17. Criterios para la selección de artículos de la temática de su interés.
$\square$ Publicados en revistas con alto factor de impacto y/o prestigio.
$\square$ Publicados en mis revistas preferidas.
$\square$ Reconocimiento de sus autores.
$\square$ Objetivos y metodología empleados.
$\square$ Resultados obtenidos.
$\square$ Otros:

18. Antigüedad de los artículos seleccionados.

$\square$ De dos o menos años de antigüedad.

$\square$ De cinco o menos años de antigüedad.

$\square$ En ocasiones consulto artículos de una antigüedad superior a cinco años.

$\square$ Otros: 
19. Hábitos de lectura de artículos.

$\square$ Leo más artículos que hace unos años.

$\square$ Leo menos artículos que hace unos años.

$\square$ Leo los documentos que me interesan en pantalla.

$\square$ Leo los artículos impresos.

$\square$ La lectura de los artículos que realizo es menos detallada que antes por la abundancia de contenidos de que dispongo.

20. Utilización de gestores bibliográficos.

$$
\begin{aligned}
& \square \text { RefWorks } \\
& \square \text { EndNote }
\end{aligned}
$$

$\square$ Otrosgestores:

$\square$ No utilizo gestores.

21. Preferencia por distribuidor de revistas electrónicas.
$\square$ Emerald
$\square$ Science de Elsevier
$\square$ Springer
$\square$ Wiley
$\square$ Otros:

22. Número de títulos de revista de consulta habitual.

$\square$ De 1 a 5.

$\square$ De 6 a 10.

$\square$ De 11 a 20 .

$\square$ Más de 20 .

23. Mencione los títulos que más utiliza (indique un máximo de cinco).

24. Mencione los títulos en que publica con mayor frecuencia los resultados de su investigación (indique un máximo de cinco).

Sólo para los investigadores que no tienen hábito de utilización de las revistas electrónicas.

25. Razones por las que no hace uso de las revistas electrónicas.

$\square$ Desconocimiento de la oferta de contenidos digitales disponible.

$\square$ Satisfacción con la colección disponible en papel en mi departamento.

$\square$ Dificultades de manejo de la tecnología que me suponen una inversión de tiempo y esfuerzo que no me compensan.

$\square$ Otros:

Para finalizar escriba por favor sus comentarios o sugerencias 
Apéndice 2

Tabla I. Datos de procedencia de los académicos que recibieron/contestaron la encuesta en la Universidad de León

\begin{tabular}{|c|c|c|c|}
\hline Departamento & Áreas de conocimiento & Docentes & $\begin{array}{l}\text { Encuestas } \\
\text { recibidas }\end{array}$ \\
\hline \multirow{4}{*}{$\begin{array}{l}\text { Biodiversidad y Gestión } \\
\text { Ambiental }\end{array}$} & Antropología Física & \multirow[t]{4}{*}{50} & \multirow[t]{4}{*}{23} \\
\hline & Botánica & & \\
\hline & Ecología & & \\
\hline & Zoología & & \\
\hline \multirow[t]{4}{*}{ Biología Molecular } & Biología Celular & \multirow[t]{4}{*}{58} & \multirow[t]{4}{*}{19} \\
\hline & Bioquímica y Biología Molecular & & \\
\hline & Genética & & \\
\hline & Microbiología & & \\
\hline \multirow[t]{4}{*}{ Ciencias Biomédicas } & Farmacología & \multirow[t]{4}{*}{49} & \multirow[t]{4}{*}{10} \\
\hline & Fisiología & & \\
\hline & Medicina Preventiva y Salud Pública & & \\
\hline & Toxicología & & \\
\hline \multirow{3}{*}{$\begin{array}{l}\text { Dirección y Economía de la } \\
\text { Empresa }\end{array}$} & Comercialización e Investigación de Mercados & \multirow[t]{3}{*}{74} & \multirow[t]{3}{*}{9} \\
\hline & Economía Financiera y Contabilidad & & \\
\hline & Organización de Empresas & & \\
\hline \multirow[t]{4}{*}{ Economía y Estadística } & Economía Aplicada & \multirow[t]{4}{*}{37} & \multirow[t]{4}{*}{2} \\
\hline & Estadística e Investigación Operativa & & \\
\hline & Fundamentos de Análisis Económico & & \\
\hline & Historia e Instituciones Económicas & & \\
\hline \multirow[t]{7}{*}{ Filología Hispánica y Clásica } & Didáctica de la Lengua y la Literatura & \multirow[t]{7}{*}{35} & \multirow[t]{7}{*}{4} \\
\hline & Filología Griega & & \\
\hline & Filología Latina & & \\
\hline & Lengua Española & & \\
\hline & Lingüística General & & \\
\hline & Literatura Española & & \\
\hline & Teoría de la Literatura y Literatura Comparada & & \\
\hline \multirow[t]{3}{*}{ Filología Moderna } & Filología Francesa & \multirow[t]{3}{*}{35} & \multirow[t]{3}{*}{3} \\
\hline & Filología Inglesa & & \\
\hline & Traducción e Interpretación & & \\
\hline \multirow[t]{5}{*}{ Geografía y Geología } & Análisis Geográfico Regional & \multirow[t]{5}{*}{23} & \multirow[t]{5}{*}{4} \\
\hline & Geodinámica Externa & & \\
\hline & Geografía Física & & \\
\hline & Geografía Humana & & \\
\hline & Paleontología & & \\
\hline \multirow{2}{*}{$\begin{array}{l}\text { Higiene y Tecnología de los } \\
\text { Alimentos }\end{array}$} & Nutrición y Bromatología & \multirow[t]{2}{*}{24} & \multirow[t]{2}{*}{7} \\
\hline & Tecnología de los Alimentos & & \\
\hline Historia & Antropología Social & 25 & 5 \\
\hline & Arqueología & & \\
\hline & Historia Antigua & & \\
\hline
\end{tabular}




\begin{tabular}{|c|c|c|c|}
\hline & Historia Contemporánea & & \\
\hline & Historia de América & & \\
\hline & Historia Medieval & & \\
\hline & Historia Moderna & & \\
\hline & Prehistoria & & \\
\hline \multirow{5}{*}{$\begin{array}{l}\text { Ingeniería y Ciencias } \\
\text { agrarias }\end{array}$} & Economía, Sociología y Política Agraria & \multirow[t]{5}{*}{25} & \multirow[t]{5}{*}{9} \\
\hline & Edafología y Química Agrícola & & \\
\hline & Fisiología Vegetal & & \\
\hline & Ingeniería Agroforestal & & \\
\hline & Producción Vegetal & & \\
\hline \multirow{2}{*}{$\begin{array}{l}\text { Medicina, Cirugía y } \\
\text { Anatomía Veterinaria }\end{array}$} & Anatomía y Anatomía Patológicas Comparadas & \multirow[t]{2}{*}{30} & \multirow[t]{2}{*}{6} \\
\hline & Medicina y Cirugía Animal & & \\
\hline \multirow{3}{*}{$\begin{array}{l}\text { Patrimonio Artístico y } \\
\text { Documental }\end{array}$} & Biblioteconomía y Documentación & \multirow[t]{3}{*}{27} & \multirow[t]{3}{*}{15} \\
\hline & Ciencias y Técnicas Historiográficas & & \\
\hline & Historia del Arte & & \\
\hline Producción Animal & Producción Animal & 31 & 2 \\
\hline \multirow{8}{*}{$\begin{array}{l}\text { Psicología, Sociología y } \\
\text { Filosofía }\end{array}$} & Filosofía & \multirow[t]{8}{*}{39} & \multirow[t]{8}{*}{9} \\
\hline & $\begin{array}{l}\text { Historia del Pensamiento y Movimientos Socia- } \\
\text { les y Políticos }\end{array}$ & & \\
\hline & Lógica y Filosofía de la Ciencia & & \\
\hline & $\begin{array}{l}\text { Métodos de Investigación y Diagnóstico en Edu- } \\
\text { cación }\end{array}$ & & \\
\hline & $\begin{array}{l}\text { Personalidad, Evaluación y Tratamiento Psico- } \\
\text { lógico }\end{array}$ & & \\
\hline & Psicología Evolutiva y de la Educación & & \\
\hline & Psicología Social & & \\
\hline & Sociología & & \\
\hline \multirow[t]{4}{*}{ Química y Física Aplicadas } & Física Aplicada & \multirow[t]{4}{*}{52} & \multirow[t]{4}{*}{5} \\
\hline & Ingeniería Química & & \\
\hline & Química Analítica & & \\
\hline & Química Física & & \\
\hline \multirow[t]{2}{*}{ Sanidad Animal } & Inmunología & \multirow[t]{2}{*}{29} & \multirow[t]{2}{*}{3} \\
\hline & Sanidad Animal & & \\
\hline
\end{tabular}

Tabla II. Ratios de descargas de artículos por investigador en la Universidad de León

\begin{tabular}{|l|c|c|c|c|c|}
\hline \multicolumn{1}{|c|}{ Años } & 2006 & 2007 & 2008 & 2009 & 2010 \\
\hline Total profesores & 986 & 1.038 & 1.045 & 1.001 & 908 \\
\hline Total descargas & 73.218 & 80.214 & 125.337 & 74.152 & 100.312 \\
\hline Ratio por profesor & 76.66 & 84.43 & 126.85 & 67.84 & 111.47 \\
\hline
\end{tabular}


Tabla III. Descarga de artículos completos en la Universidad de León

\begin{tabular}{|l|c|c|c|c|c|}
\hline \multicolumn{1}{|c|}{ Distribuidor } & 2006 & 2007 & 2008 & 2009 & 2010 \\
\hline ScienceDirect & 66825 & 68491 & 110027 & 62121 & 77778 \\
\hline SpringerLink & 3063 & 7651 & 10381 & 7349 & 10792 \\
\hline Wiley & 3330 & 4072 & 4929 & 4682 & 11742 \\
\hline
\end{tabular}

Tabla IV. Revistas de uso y comunicación científica preferente

\begin{tabular}{|c|c|c|c|c|c|}
\hline $\begin{array}{l}\text { Revistas de uso } \\
\text { preferente }\end{array}$ & $\begin{array}{c}\text { Revistas preferidas } \\
\text { para publicar }\end{array}$ & Editor/Distribuidor & $\begin{array}{c}\text { JCR } \\
5 \text {-Year }\end{array}$ & SJR 2011 & Materia \\
\hline $\begin{array}{l}\text { Anuario del } \\
\text { Departamento } \\
\text { de Historia y } \\
\text { Teoría del Arte }\end{array}$ & & $\begin{array}{l}\text { Universidad Autónoma } \\
\text { de Madrid }\end{array}$ & & & - History of Art \\
\hline \multirow[t]{3}{*}{$\begin{array}{l}\text { Applied and } \\
\text { Environmental } \\
\text { Microbiology }\end{array}$} & & $\begin{array}{l}\text { American Society for } \\
\text { Microbiology }\end{array}$ & 4.529 & 0.306 & $\begin{array}{l}\text { Biotechnology \& } \\
\text { Applied Micro- } \\
\text { biology } \\
\text { - Microbiology }\end{array}$ \\
\hline & $\begin{array}{l}\text { Atmospheric } \\
\text { Research }\end{array}$ & ScienceDirect & 1.743 & 0.088 & $\begin{array}{l}\text { - Meteorology \& } \\
\text { Atmospheric } \\
\text { Sciences } \\
\end{array}$ \\
\hline & $\begin{array}{l}\text { Ecological Engi- } \\
\text { neering }\end{array}$ & ScienceDirect & 2.807 & 0.100 & $\begin{array}{l}\cdot \text { Ecology } \\
\cdot \text { Engineering } \\
\text { Environmental } \\
\cdot \text { Environmental } \\
\text { Sciences }\end{array}$ \\
\hline Ecology & & $\begin{array}{l}\text { Ecological Society } \\
\text { of America }\end{array}$ & 6.218 & 0.315 & - Ecology \\
\hline $\begin{array}{l}\text { Hispania: Revista } \\
\text { Española de } \\
\text { Historia }\end{array}$ & & $\begin{array}{l}\text { Consejo Superior } \\
\text { de Investigaciones } \\
\text { Científicas }\end{array}$ & & & - History \\
\hline $\begin{array}{l}\text { El Profesional de } \\
\text { la Información }\end{array}$ & \begin{tabular}{|l|} 
El Profesional de la \\
Información
\end{tabular} & EPI & $0.375^{\star}$ & 0.027 & $\begin{array}{l}\text { - Information } \\
\text { Science \& Library } \\
\text { Science }\end{array}$ \\
\hline $\begin{array}{l}\text { Food Microbio- } \\
\text { logy }\end{array}$ & Food Microbiology & ScienceDirect & 3.473 & 0.135 & $\begin{array}{l}\text { - Biotechnology } \\
\text { \& Applied Microbio- } \\
\text { logy } \\
\text { - Food Science \& } \\
\text { Technology } \\
\text { - Microbiology }\end{array}$ \\
\hline $\begin{array}{l}\text { Forest Ecology } \\
\text { and Manage- } \\
\text { ment }\end{array}$ & $\begin{array}{l}\text { Forest Ecology and } \\
\text { Management }\end{array}$ & ScienceDirect & 2.507 & 0.080 & - Forestry \\
\hline Hepatology & & Wiley & 11.044 & 1.278 & $\begin{array}{l}\text { - Gastroenterology \& } \\
\text { Hepatology }\end{array}$ \\
\hline
\end{tabular}




\begin{tabular}{|c|c|c|c|c|c|}
\hline $\begin{array}{l}\text { International } \\
\text { Journal of } \\
\text { Climatology }\end{array}$ & & Wiley & 3.913 & 0.133 & $\begin{array}{l}\text { - Meteorology \& } \\
\text { Atmospheric } \\
\text { Sciences }\end{array}$ \\
\hline $\begin{array}{l}\text { International } \\
\text { Journal of Food } \\
\text { Microbiology }\end{array}$ & $\begin{array}{l}\text { International } \\
\text { Journal of Food } \\
\text { Microbiology }\end{array}$ & ScienceDirect & 3.557 & 0.150 & $\begin{array}{l}\text { - Food Science \& } \\
\text { Technology } \\
\text { - Microbiology }\end{array}$ \\
\hline \multirow[t]{2}{*}{$\begin{array}{l}\text { Journal of } \\
\text { Applied } \\
\text { Microbiology }\end{array}$} & $\begin{array}{l}\text { Journal of Applied } \\
\text { Microbiology }\end{array}$ & Wiley & 2.798 & 0.135 & $\begin{array}{l}\text { - Biotechnology } \\
\text { \&Applied Microbio- } \\
\text { logy } \\
\text { - Microbiology }\end{array}$ \\
\hline & $\begin{array}{l}\text { Journal of Biologi- } \\
\text { cal Chemistry }\end{array}$ & $\begin{array}{l}\text { American Society } \\
\text { for Biochemistry and } \\
\text { Molecular Biology }\end{array}$ & 5.498 & & $\begin{array}{l}\text { - Biochemistry \& } \\
\text { Molecular Biology }\end{array}$ \\
\hline $\begin{array}{l}\text { Journal of } \\
\text { Documentation }\end{array}$ & & Emerald & 1.407 & 0.046 & $\begin{array}{l}\text { - Information } \\
\text { Science \& Library } \\
\text { Science }\end{array}$ \\
\hline $\begin{array}{l}\text { Journal of Food } \\
\text { Protection }\end{array}$ & $\begin{array}{l}\text { Journal of Food } \\
\text { Protection }\end{array}$ & $\begin{array}{l}\text { International } \\
\text { Association for Food } \\
\text { Protection }\end{array}$ & 2.001 & 0.109 & $\begin{array}{l}\text { - Biotechnology } \\
\text { \&Applied Microbio- } \\
\text { logy } \\
\text { - Food Science \& } \\
\text { Technology }\end{array}$ \\
\hline \multirow[t]{2}{*}{$\begin{array}{l}\text { Journal of } \\
\text { Hepatology }\end{array}$} & & ScienceDirect & 7.404 & 0.765 & $\begin{array}{l}\text { - Gastroenterology \& } \\
\text { Hepatology }\end{array}$ \\
\hline & $\begin{array}{l}\text { Journal of Pineal } \\
\text { Research }\end{array}$ & Wiley & 4.823 & 0.244 & $\begin{array}{l}\text { - Endocrinology \& } \\
\text { Metabolism } \\
\text { - Neurosciences } \\
\text { - Physiology }\end{array}$ \\
\hline \multirow[t]{4}{*}{ Meat Science } & & ScienceDirect & 3.000 & 0.095 & $\begin{array}{l}\text { - Food Science \& } \\
\text { Technology }\end{array}$ \\
\hline & Molecular Plant & $\begin{array}{l}\text { Oxford University } \\
\text { Press }\end{array}$ & 4.307 & 0.718 & $\begin{array}{l}\text { - Biochemistry \& } \\
\text { Molecular Biology } \\
\text { - Plant Sciences }\end{array}$ \\
\hline & $\begin{array}{l}\text { Physiologia } \\
\text { Plantarum }\end{array}$ & Wiley & 2.947 & 0.240 & - Plant Sciences \\
\hline & Phytochemistry & ScienceDirect & 3.335 & 0.173 & - Plant Sciences \\
\hline Plant Ecology & Plant Ecology & Springer & 2.184 & 0.075 & $\begin{array}{l}\text { - Plant Sciences } \\
\text { - Ecology } \\
\text { - Forestry }\end{array}$ \\
\hline Plant Physiology & & $\begin{array}{l}\text { American Society of } \\
\text { Plant Biologists }\end{array}$ & 7.016 & 0.739 & - Plant Sciences \\
\hline Planta & & Springer & 3.451 & 0.243 & - Plant Sciences \\
\hline Psicothema & & $\begin{array}{l}\text { Colegio Oficial de Psi- } \\
\text { cólogos de Asturias }\end{array}$ & 1.018 & 0.040 & $\begin{array}{l}\text { - Psychology, Multi- } \\
\text { disciplinary }\end{array}$ \\
\hline $\begin{array}{l}\text { Revista Española } \\
\text { de Documenta- } \\
\text { ción Científica }\end{array}$ & & $\begin{array}{l}\text { Consejo Superior } \\
\text { de Investigaciones } \\
\text { Científicas }\end{array}$ & $0.489^{\star}$ & 0.031 & $\begin{array}{l}\text { - Information } \\
\text { Science \& Library } \\
\text { Science }\end{array}$ \\
\hline
\end{tabular}




\begin{tabular}{|c|c|c|c|c|c|}
\hline & Serials Review & ScienceDirect & 0.582 & 0.033 & $\begin{array}{l}\text { - Information } \\
\text { Science \& Library } \\
\text { Science }\end{array}$ \\
\hline & Theriogenology & ScienceDirect & 2.506 & 0.081 & \begin{tabular}{|l|}
$\cdot$ - Reproductive \\
Biology \\
- Veterinary Sciences
\end{tabular} \\
\hline
\end{tabular}

*Factor de impacto del año 2010

$\infty$ 